\title{
Prioritized Static Slicing and its Application to Fault Localization
}

\author{
Yiji Zhang*, Raul Santelices \\ University of Notre Dame, Indiana, USA
}

\begin{abstract}
Static slicing is a popular program analysis used in software engineering to find which parts of a program affect other parts. Unfortunately, static slicing often produces large and imprecise results because of its conservative nature. Dynamic slicing can be an alternative in some cases, but it requires detailed runtime information that can be hard or impossible to obtain or re-create. This is often the case when users report bugs in deployed software. In this paper, we significantly improve the precision of static slicing through PRIOSLICE, a novel technique that exploits the insight that not all statements in a static slice are equally likely to affect another statement such as a failing point. PRIOSLICE first computes a probabilistic model of the dependencies in the program. In this model, some data dependencies are more likely to occur than others and control dependencies are less likely than data dependencies to propagate effects (e.g., errors). PRIOSLICE then traverses the program backwards, like static slicing, but in an order defined by the computed dependence probabilities. Our study of fault localization on various Java subjects indicates that PRIOSLICE can help localize faults much more effectively than existing static-slicing approaches.
\end{abstract}

Keywords: static slicing, probabilistic slicing, thin slicing, dependence analysis, fault localization, program analysis

\footnotetext{
${ }^{*}$ Corresponding author. Tel: +15748550201

Email addresses: yzhang20@nd. edu (Yiji Zhang), rsanteli@nd.edu (Raul Santelices)
} 


\section{Introduction}

Program slicing is a popular program-analysis technique used for many softwareengineering tasks, such as fault localization and program comprehension [1]. Static program slicing, in particular, finds the set of all statements in the program-the slice - that might affect a particular point in that program. Unfortunately, however, static slicing is often too imprecise to be practical because it tends to produce slices whose sizes are very large [2,3], even when considering calling contexts for interprocedural analysis [4].

To reduce the size of slices and, thus, increase their usefulness, researchers have developed other forms of program slicing such as dynamic slicing [5,6], variants of it $[7,8,9]$, combinations of static slices with execution data $[10,11,12$, 13], and slice pruning based on various criteria [14, 15, 16, 17]. These variants, however, focus on subsets of all program behaviors and can miss faulty code. Moreover, these techniques can still be imprecise [3].

Dynamic approaches provide concrete insights on how programs behave in typical cases, provided that a representative set of executions is used. Unfortunately, such a set of executions is not always available to developers. For fault localization, one failed execution can be enough to find a fault, but multiple executions are often needed to make localization effective $[18,19]$. In many cases, such as bugs reported by users, developers may not even have one execution to work with. Another problem is that faulty executions can be hard to reproduce for debugging. This problem is exacerbated by non-determinism in software whose behavior depends on external factors such as time and thread interleaving. One possibility is to deploy software instrumented to collect dynamic slices, but this would incur unacceptable runtime overheads. Therefore, when dynamic slicing is not an option, static slicing must be used.

In this paper, we present a novel technique called PRIOSLICE which considerably increases the effectiveness of static slicing by reducing the negative effects of its imprecision. PRIOSLICE prioritizes the inspection of a static slice (i.e., the subset of the program that affects another program point) using a probabilistic model of how program dependencies $[20,21]$ (the building blocks of program slices) occur and how they affect the slicing criterion (i.e., a value in a program point from which the slice is computed). To that end, for each statement in a slice, PRIOSLICE computes a weight in the range $[0,1]$ representing the likelihood that

this statement belongs to the slice. Thus, PRIOSLICE tells not only whether a statement is in a slice, but also how much.

The probabilistic model of PRIOSLICE exploits two key insights. The first 
insight is that not all data dependencies [20] are equally likely to occur because of control-flow and aliasing [22] reasons. Data dependencies are treated (mostly) as equals by existing static-slicing techniques $[1,16]$. Our model and technique, in contrast, gives a greater priority to the data dependencies that are most likely to occur and cause a particular program behavior (e.g., a failure) and, thus, more quickly identifies its causes (e.g., a fault).

The second insight used in this model is that control dependencies [21] are usually weaker than data dependencies at propagating effects such as errors [23, $3,16]$. Therefore, they should not be treated as equals with data dependencies for slice inspection, as Weiser's method does [1], but they should not be discarded upfront either as, for example, thin slicing does [16]. Our model incorporates both types of dependencies while giving them different weights according to their respective effect-propagation potential estimated by our probabilistic analysis.

Concretely, PRIOSLICE first computes the static backward slice from a slicing criterion in a program and then solves the system of equations given by the model for the dependence graph of that program. The solution for these equations is the set of weights for the statements in the static slice. These weights are the result of using a standard method for solving real-valued data-flow problems [24]. PRIOSLICE uses these results to perform a best-first traversal [25] of the slice, which replaces Weiser's breadth-first traversal approach [1] of static dependencies by prioritizing statements according to their weight.

Unlike statistical approaches (e.g., [19]) which provide seemingly-random orders to inspect programs [26], static-slicing approaches such as PRIOSLICE navigate the dependence graph in order by visiting only neighbors of visited nodes as suggested by Weiser [1]. Each new statement to inspect is reached via a direct dependence to another statement already inspected. Navigating dependencies is, thus, a more natural way for developers to inspect programs [26].

To study the feasibility and effectiveness of PRIOSLICE, in this paper, we chose fault localization as the application-arguably the most important one. Fault localization is the first step for debugging a program, in which the causes of a reported failure must be found before the program can be fixed. We implemented our technique for slicing Java-bytecode programs using our dependenceanalysis infrastructure DUA-FORENSICS [27, 28]. Then, we applied three staticslicing techniques to several faults across various Java subjects. These techniques are PRIOSLICE, Weiser's traversal approach for static slicing [1], and thin slicing [16].

Our results for these subjects and faults indicate with statistical significance that PRIOSLICE can reach the faulty code faster than static slicing. PRIOSLICE 
traverses, on average, about $13 \%$ of the program, whereas Weiser's approach requires an average inspection of about $27 \%$ of the program to find each fault. Meanwhile, thin slicing, when it was able to find a fault at all (only 12 out of 60 in total), ${ }^{1}$ also required to inspect about $18 \%$ of the program on average. For those faults, PRIOSLICE required about the same amount of program (i.e. 17\%) to be inspected on average. Though we can not draw the conclusion that PRIOSLICE is significantly better than thin slicing, we also can't tell that thin slicing is different from PRIOSLICE. (Not to mention that PRIOSLICE was applicable to all faults, whereas thin slicing was only able to $20 \%$ of the faults.)

The most important benefit of this work is that it provides a new way of looking at static slices in which statements are distinguished by relevance rather than just by membership in the slice. This feature makes PRIOSLICE improve fault localization considerably. Its focused traversal of dependence graphs also hints at its potential for related applications such as program comprehension. We also applied a preliminary forward version of this model [29, 30] for change-impact analysis with promising first results. Finally, the probabilistic model underlying PRIOSLICE still has room for improvements so that its benefits can further increase in the future.

In all, the contributions of this paper include:

- A new form of static program slicing that indicates not only whether, but also how much, each statement belongs to a slice.

- A new technique, PRIOSLICE that realizes this concept of probabilistic slicing to prioritize the traversal of slices by the estimated degree of membership of their statements.

- A study indicating that PRIOSLICE can considerably reduce the effort of static fault localization with respect to Weiser's original approach and thin slicing.

\section{Background}

This section presents core concepts needed for this paper and illustrates those concepts using the example program of Figure 1.

\subsection{Program Dependencies}

Control and data dependencies are the building blocks of program slicing [1, 4]. A statement $T$ is control dependent [21] on a statement $S$, denoted $(S, T)$,

\footnotetext{
${ }^{1}$ Control dependencies can be added for the remaining faults after data dependencies are exhausted, but the order in which this is done is unclear [16].
} 


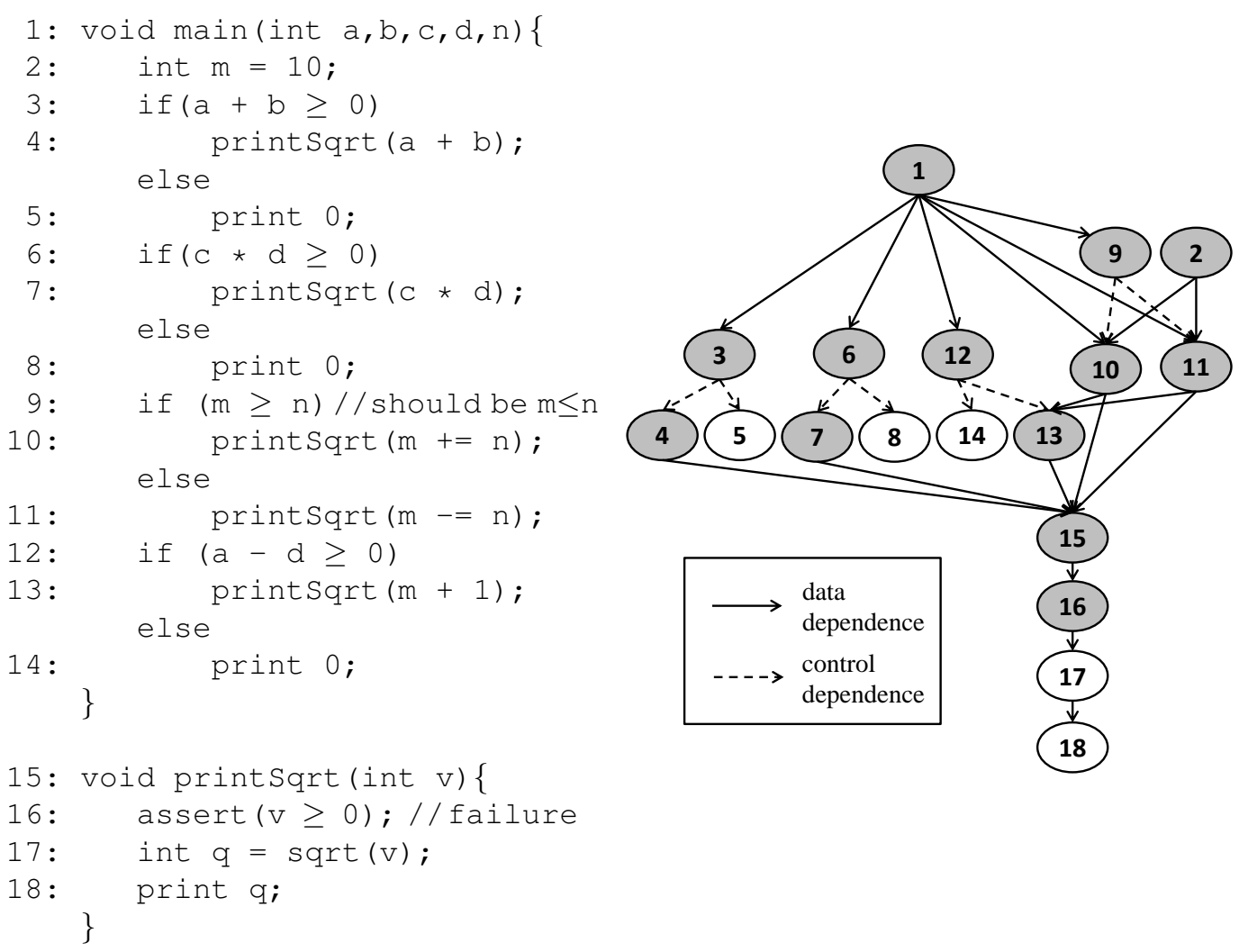

Figure 1: Example program used for illustration and its dependence graph on the right.

if a control-flow (jump) decision taken at $S$ determines whether $T$ is necessarily executed. For example, in Figure 1, line 4 is control dependent on line 3 because the decision made at 3 determines whether 4 is necessarily executed.

A statement $U$ is data dependent [20] on a statement $D$ if a variable $v$ can be defined (written) at $D$ and used (read) at $U$ and there is a definition-clear path in the program for $v$ (i.e., a path that does not re-define $v$ ) from $D$ to $U$. This definition of data dependence implies that, if $v$ is accessed via a pointer or reference $p$, $p$ points to the same memory location at $D$ and $U$. We denote a data dependence of $U$ on $D$ for variable $v$ as $(D, U, v)$, or just $(D, U)$.

\subsection{Program Slicing}

Program slicing was originally developed by Weiser [1]. A static slice for a set of variables $V$ at a program point $C$ - the slicing criterion-is the subset of all program statements that affect those values at that point. A slice can also be 
defined simply for a program point $C$ as the set of all statements that affect either the values used at $C$ or the execution of $C$.

To realize Weiser's approach, we use the dependence graph of the program. This graph is using the data and control dependencies reachable backwards from the slicing criterion, where nodes are statements and edges are dependencies. Weiser's approach performs a backward breadth-first traversal of this graph to produce a visit order of the statements. For fault localization, this order can be used to measure the effort required to find a fault - the number of nodes traversed until the fault is reached.

To illustrate, consider the example program in Figure 1. Suppose that we want to determine all statements in this program that affect the value of $v$ at statement 16. Using the backward transitive closure of control and data dependencies from that statement, we obtain the backward static slice $\langle 1,2,3,4,6,7,9,10,11$, $12,13\rangle$.

Of all variants of static slicing proposed afterward, thin slicing [16] is the one used in this paper. Thin slicing excludes control dependencies at first to focus on data dependencies. It also excludes data dependencies for pointers or references used in definitions of other variables and control decisions. The traversal of the included statements is also done backwards in breadth-first order. Naturally, a goal (e.g., a fault) might be missed in this traversal. Thus, dependencies initially excluded can be added incrementally as needed [16] although the visit order for them is unclear.

\section{Data-Dependence Model}

In preliminary work [31], we proposed a technique called PRIODU to estimate the execution likelihood of data dependencies in a program. (Our final goal of prioritizing slice inspections, stated in that work, is finally realized now with PRIOSLICE.) For PRIODU, we used control-flow and points-to analysis to estimate the likelihood of occurrence of each data dependence. Our insight is that not all data dependencies are equally likely to occur at runtime.

In this section, we present the aspects of PRIODU that we use for data dependencies in our new technique, PRIOSLICE. (In Section 4, we will expand this to include control dependencies and entire dependence graphs, all of which define PRIOSLICE.) 


\subsection{Reaching Probability}

A key part of PRIODU is the concept of reaching probability. For statements $a$ and $b$, the event $a \rightarrow_{R} b$ states that the program follows some path from $a$ to $b$. The probability of $a \rightarrow_{R} b$ is:

$$
P\left(a \rightarrow_{R} b\right)= \begin{cases}1 & \text { if } a=b ; \\ P\left(a \rightarrow_{R} b \mid a \neq b\right) & \text { otherwise }\end{cases}
$$

If $a$ and $b$ are the same statement, the reaching probability is trivially 1 . Otherwise, if $a$ is different from $b, P\left(a \rightarrow_{R} b\right)$ is the probability of reaching $b$ from $a$ through some successor of $a$ :

$$
\begin{gathered}
P\left(a \rightarrow_{R} b \mid a \neq b\right)=P\left(\bigvee_{s \in} a \rightarrow_{S} s \wedge s \rightarrow_{R} b\right) \\
=\sum_{s \in \operatorname{succ}(a)} P\left(a \rightarrow_{S} s\right) \times P\left(s \rightarrow_{R} b\right)
\end{gathered}
$$

where $\operatorname{succ}(a)$ is the set of all control-flow successors of statement $a$ and $a \rightarrow_{S} s$ states that $s$ is the successor that executes right after $a$. This probability is the sum of the probabilities of reaching $b$ through the successors of $s$ because the events $\left(a \rightarrow_{S} s \wedge s \rightarrow_{R} b\right)$ in the disjunction are exclusive - exactly one statement succeeds another in an execution. Also, the two events in each term of the disjunction are independent, so their joint probability is the product of their respective probabilities. Note that the probability of the second event, $s \rightarrow_{R} b$, can make Equation 2 recursive. Thus, approaches for solving real-valued data-flow problems must be used [24]. The probability of $a \rightarrow_{S} s$, specifically, is given by a branch prediction mechanism (a parameter of our technique). The only constraint is

$$
\sum_{s \in \operatorname{succ}(a)} P\left(a \rightarrow_{S} s\right)=1
$$

\subsection{Propagation Probability}

The other building block of PRIODU we use in PRIOSLICE is the propagation probability of data dependencies. Without loss of generality, we assume that the program defines at most one variable per statement. A data dependence $(d, u)$ propagates an effect (e.g., an error) if (1) the statement $u$ might use the variable $v$ defined at $d$ and $u$ is reached from $d$ through a definition-clear path for $v$, and 
(2) the memory location $v$ defined at $d$ and used at $u$ is the same. We denote these two events as $d \rightarrow_{R U_{v}} u$ and $\operatorname{alias}(d, u, v)$, respectively. Our model defines the propagation probability of $(d, u)$ as

$$
\begin{array}{r}
P\left(d \stackrel{d d}{\rightarrow}_{p} u\right)=P\left(d \rightarrow_{R U_{v}} u \wedge \operatorname{alias}(d, u, v)\right) \\
=P\left(d \rightarrow_{R U_{v}} u\right) \times P(\operatorname{alias}(d, u, v))
\end{array}
$$

where the alias event might dependent on the path that reaches $u$ from $d$. However, computing the probability of these two events as dependent events is impractical because of the large or even infinite number of paths from $d$ to $u$ that do not redefine $v$. For this reason, to make the model tractable, we treat these two events as independent and, thus, simply multiply their respective probabilities.

The probability of the first of those two events on the right-hand side of Equation 4 is computed using the following formula:

$$
P\left(d \rightarrow_{R U_{v}} u\right)=P\left(\begin{array}{ll}
\bigvee & d \rightarrow_{S} s \wedge \\
s \in & (s=u \vee \\
\operatorname{succ}(d) & \left.\left(u \in \operatorname{ru}(s, v) \wedge s \rightarrow_{R U_{v}} u\right)\right)
\end{array}\right)
$$

where $r u(s, v)$ is the set of reachable uses [20] of $v$, the variable defined at $d$, from statement $s$. Equation 5 states that the probability that a use of a variable $v$ in a statement $u$ is reachable from a definition of $v$ in statement $d$ through a definitionclear path for $v$ is the probability that this happens via some control-flow successor of $d$ (i.e., one of the statements that can execute right after $d$ ). The events that $s$ is the statement executed after $d$ (i.e., $d \rightarrow_{S} s$ ) and that either $u$ is $s$ or $u$ is reachable from $s$ (i.e., $\left.s=u \vee\left(u \in r u(s) \wedge s \rightarrow_{R U_{v}} u\right)\right)$ are independent from each other and their conjunction is also independent from the events for all other successors of $d$. Hence, this probability can be decomposed into a sum of the products of the probabilities of these events, similar to Equation 2. The term $s \rightarrow_{R U_{v}} u$ makes the computation of the propagation probability for a data dependence also a recursive problem.

The second term of Equation 4 is the event that the defined and used memory locations are the same at runtime - that the variable $v$ is the same at the definition and use. Its probability is one if $v$ is accessed directly but may be less than one if it is accessed via a base pointer (e.g., $p . f$ ) that may point to either $v$ or some other variable. Our technique uses points-to sets [22] to represent memory locations statically. It estimates the aliasing probability as the probability that any element from one points-to set is also in the other set, which corresponds to the Jaccard similarity [32] of those sets: 


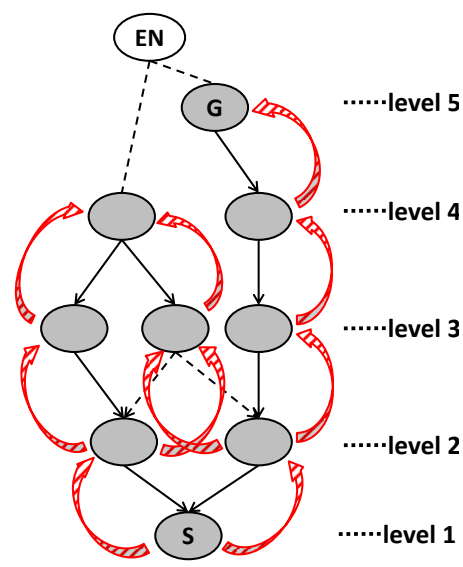

(a) Weiser's Slicing

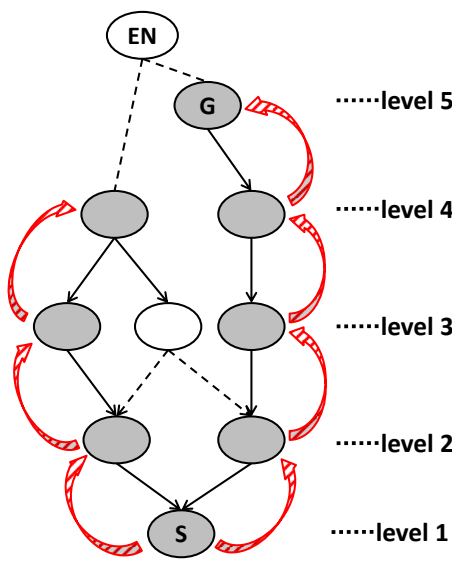

(b) Thin Slicing

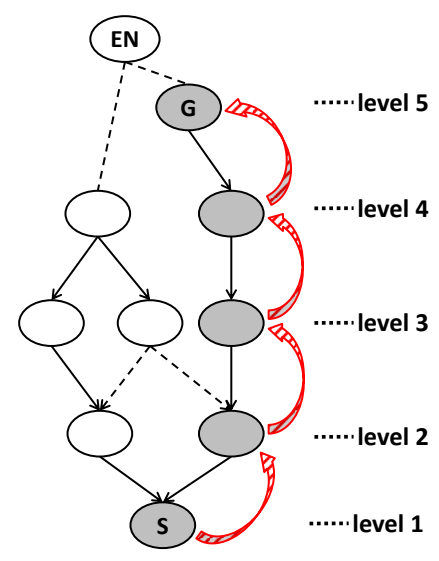

(c) PrioSlice

data dependence ----> control dependence ----- path

Figure 2: Intuition behind PRIOSLICE: traversal of dependence graphs guided by node importance

$$
P(\operatorname{alias}(d, u, v))=\frac{|P 2 \operatorname{Set}(d, v) \bigcap \operatorname{P2Set}(u, v)|}{|\operatorname{P} 2 \operatorname{Set}(d, v) \bigcup P 2 \operatorname{Set}(u, v)|}
$$

where $P 2 \operatorname{Set}(s, v)$ is the set of all variables that might be aliased with $v$ at $s$, including $v$. If $v$ is accessed directly (i.e., not through a pointer) at $s$, then $P 2 \operatorname{Set}(s, v)$ is trivially $\{v\}$.

\section{Technique}

This section presents our new technique, PRIOSLICE, which builds on the concepts of Sections 2 and 3. We explain the intuition of the technique, give a detailed overview, and then formally present the model and algorithm.

\subsection{Intuition}

Two main observations motivate PRIOSLICE:

1. Data dependencies are more likely than control dependencies to propagate effects such as faults.

2. Different data dependencies can have different probabilities of propagating those effects. 
Traditionally, slicing has not exploited these two kinds of differences. Instead, slicing simply induces a traversal of the dependence graph that weights all edges equally, even though some edges are more likely than others to lead to a goal (e.g., a fault). Thin slicing, in contrast, uses these intuitions only to some extent by ignoring at first some types of dependencies that are deemed less important, but it might miss the goal. Even if it reaches a goal, it still regards all remaining dependencies equally.

Figure 2 illustrates our intuition that, instead of treating all dependencies equally or pruning dependencies, a smarter prioritization of dependencies during the dependence-graph traversal can reach a goal faster than existing approaches. The three graphs, from left to right, represent the same part of the same dependence graph. Each node in each graph represents a statement in the program. Each graph has an entry node $E N$. To these graphs, three traversal approaches were respectively applied: Weiser's original approach, thin slicing, and PRIOSLICE.

Each traversal starts at node $S$, proceeds backwards, and ends at the goal node $G$. The shaded nodes are those visited during the traversal. The curved-up arrows indicate the steps of the traversal. The levels in the figure represent, from a breadth-first perspective, the depth of the nodes at those levels with respect to $S$ (which has distance 1 to itself). Weiser's approach-a breadth-first searchtraverses the graph level by level until it reaches the goal. Thin slicing reduces the traversal effort by one node by avoiding control dependencies (dashed edges) at first.

The rationale for PRIOSLICE, in contrast, is that the dependencies that are most likely to propagate an effect from $G$, such as those on the right in each graph, are visited first. If a traversal weighs dependencies properly, that traversal will tend to find the goal faster. We present PRIOSLICE to achieve this goal.

\subsection{Overview}

Consider the usage scenario for fault localization of Figure 3. First, a user runs a program and a failure occurs. The user then submits to the developers a bug report describing the failure. The developers examine this report and consider reproducing the failing execution to locate the causes of that failure. The report, however, might not include all inputs and conditions under which the failure occurred, or the failure might be too hard to reproduce for a variety of reasons (e.g., there is non-determinism). In that case, a technique such as static slicing or PRIOSLICE must be used, which only needs the program and the point in the program where the failure was manifested (the slicing criterion). 


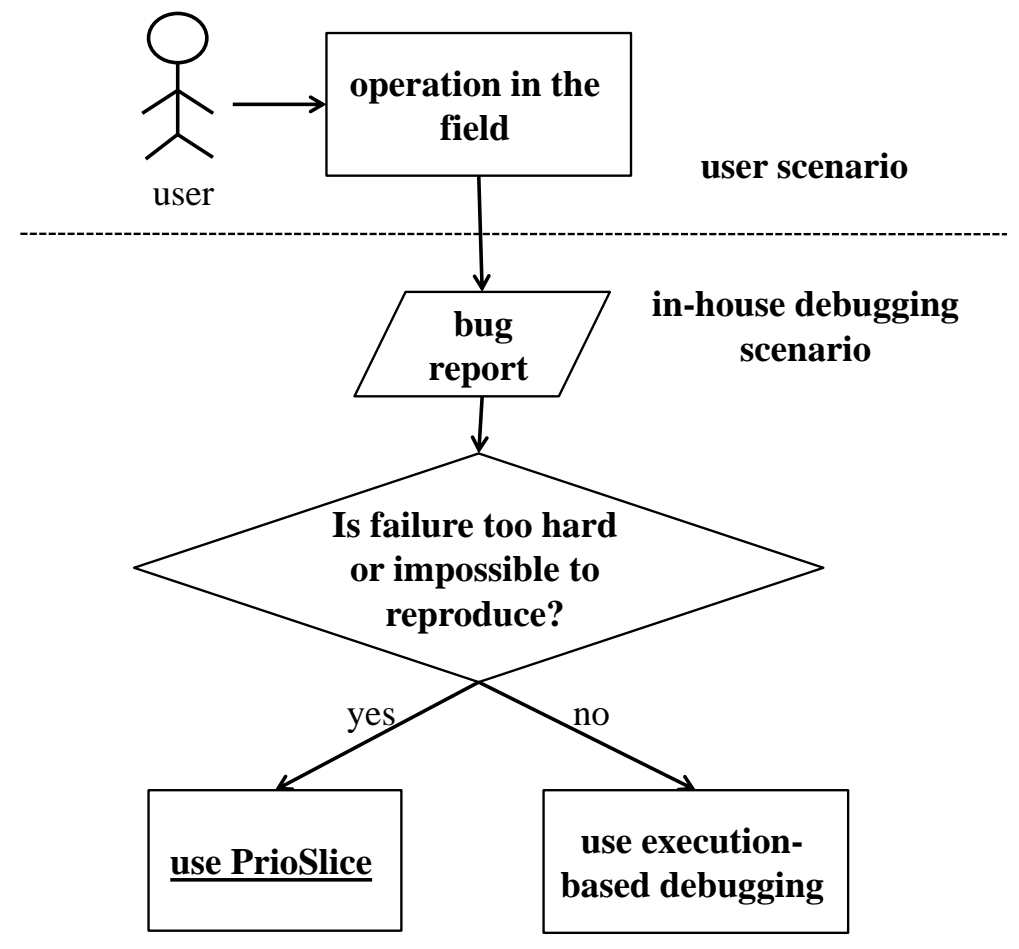

Figure 3: Static scenario for fault localization

Figure 4 shows the big picture of how PRIOSLICE works. First, a dependencegraph builder in PRIOSLICE takes a program $P$ and a slicing criterion $C$ and computes the dependence graph $G$ for the entire program. Second, PRIOSLICE computes, for each dependence $(s, t)$ in $G$, the probability that, if a fault occurs at $s$ or propagates to $s$, that fault continues propagating to $t$ via this dependence. Finally, PRIOSLICE performs a backward prioritized traversal of $G$. This traversal starts at $C$ and, in each step, takes the highest-probability dependence that links an unvisited node to a visited node and visits the unvisited (source) node of that dependence. The traversal ends when the developer finds the goal (e.g., the fault that causes the failure at $C$ ).

To illustrate, consider the example program of Figure 1. This program takes five integers as inputs and calls at different points the helper function print Sqrt depending on control-flow decisions influenced by the input values. printSqrt takes an integer and checks whether its value is zero or greater, calculates its square root, and prints the result. The example includes a fault at line 9: when the 


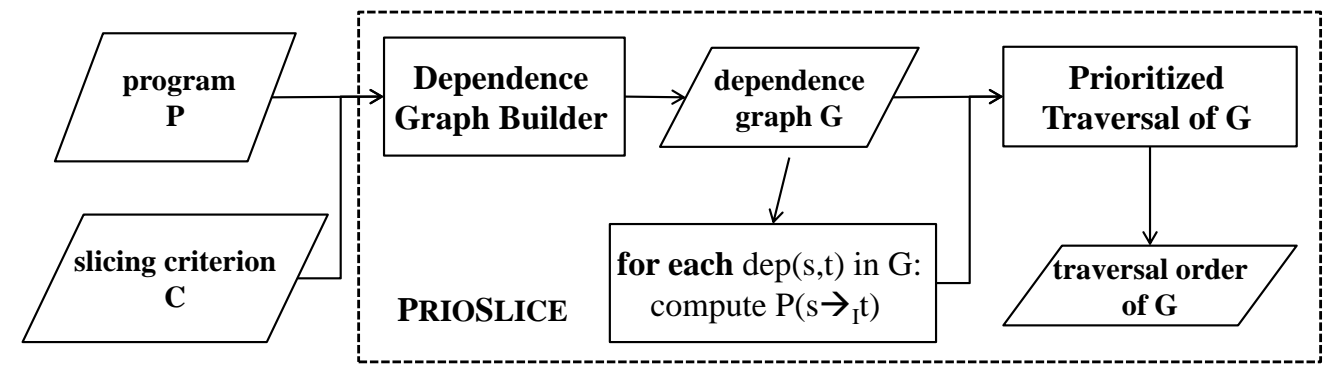

Figure 4: High-level description of the internal process of PRIOSLICE

value of $m$ is equal to input $n$ or greater, the program proceeds to line 10 to assign $m$ the possibly-negative value $m+n$, and when $m$ is less than $n$, line 11 definitely assigns $m$ a negative value. Thus, printSqrt can be called at lines 10, 11, and 13 with a negative value that makes the assertion at line 16 fail.

Figure 1 on the right shows the dependence graph for this example program to help see how line 16 is affected by other lines and, specifically, by the fault at line 9. Each node in the graph represents a line (statement) in the program ${ }^{2}$ and each edge represents a data dependence (solid edge) or control dependence (dotted edge) between two statements. For this example, the shaded nodes are the static backward slice from $v$ at line 16 (Weiser's approach).

After creating the dependence graph, PRIOSLICE solves a system of equations (described in Section 4.3) to estimate the probability of each statement in the slice of affecting the slicing criterion. Intuitively, PRIOSLICE works as follows: assuming that the developer has reached a node $t$ during inspection, node $s$ affects node $t$ through dependence $(s, t)$ to the extent (likelihood) that

1. node $s$ is reached when the program runs, and

2. a faulty state at $s$ propagates to $t$ through this dependence.

For the first event, PRIOSLICE performs a reaching-probability analysis (Section 3.1) to estimate the likelihood that a statement $s$ is reached from the entry of the program for any possible execution. For example, in Figure 1, line 15 is

\footnotetext{
${ }^{2}$ For simplicity, we omitted the customary entry and start nodes.
} 
reached if any of the conditions at lines 3, 6, 10, and 12 evaluates to true. The probability of reaching line 15 is 1.0 because either outcome of the condition at line 9 causes the helper function to be called. Each of the call sites for that function, however, has a reaching probability of less than 1.0 which corresponds to the probability that the guarding conditional takes the branch leading to that call site (e.g., 0.5).

In general, there are multiple control-flow paths between two statements in a program because of conditional statements (e.g., branches, multi-target virtual calls). The possible outcomes of each conditional statement are exclusive events, so the probability of the disjunction of outcomes is simply the sum of the individual probabilities of those outcomes. For different conditional statements, to keep our model manageable, we treat their outcomes as independent events, regardless of any correlation in reality. Therefore, the probability for a sequence of outcomes along a path is the product of the individual probabilities of those outcomes.

For the second event, PRIOSLICE first estimates, for each dependence, how likely it is that the dependence is covered (executed) after its source is reached and that this covered dependence propagates a fault from source to target. In Figure 1, for example, the probability of covering data dependence $(1,9)$ is 1.0 simply because line 9 always executes after line 1 (barring an assertion violation at line 16 in between). For this dependence, the variable defined at the source line is the same used at the target line because it is accessed directly and not via pointers, so its aliasing probability is 1.0 . Therefore, the propagation probability for dependence $(1,9)$ is 1.0 . For a less trivial example, PRIOSLICE estimates that the probability that line 1 affects line 10 via data dependence $(1,10)$ is the probability of reaching 10 via branch $9 \rightarrow 10$ (e.g., 0.4 ).

For a control dependence, the coverage probability is simply the probability of the corresponding outcome of a decision statement at the source of the dependence. For propagation, a faulty state at the source of the dependence propagates to the target only if a different decision (e.g., another branch) from the source is taken in the absence of the fault. In other words, the propagation occurs when the control decision is made by mistake. To illustrate, consider control dependence $(9,10)$ in Figure 1 . If the probability of the corresponding branch is, say, .4 , the probability that a fault causes the program to wrongly take this branch is $1-.4=.6$. Thus, the propagation probability of $(9,10)$ is the product of the probabilities of taking this branch and that taking it is a mistake: $.4 \times .6=.24$.

For statements $s$ on which the starting statement $f$ is transitively dependent (i.e., via sequences of more than one dependence), each statement $s$ affects $f$ through one or more successors of $s$. Consider, for example, line 9 in Figure 1. 
Table 1: PRIOSLICE traversal from line 16 in the example. All branch probabilities are 0.5.

\begin{tabular}{|l|l|}
\hline node visit order & affecting probabilities of discovered nodes \\
\hline$\emptyset$ & $\mathrm{P}(16)=1$ \\
\hline 16 & $\mathrm{P}(15)=1$ \\
\hline 16,15 & $\mathrm{P}(4)=\mathrm{P}(7)=\mathrm{P}(10)=\mathrm{P}(11)=\mathrm{P}(13)=1$ \\
\hline $16,15,\{4,7,10,11,13\}$ & $\mathrm{P}(3)=\mathrm{P}(6)=\mathrm{P}(12)=.25 ; \mathrm{P}(9)=.375$ \\
\hline $16,15,\{4,7,10,11,13\}, 9$ & stop: found the fault \\
\hline
\end{tabular}

The probability of this line affecting line 16 via the call at line 11 has three parts: line 9 affects line 11, the update of $m$ at line 11 affects line 15, and line 15 affects line 16. The probability of these parts are, respectively, .24, 1, and 1, for a total probability of 24 .

More generally, there can be many control-flow paths along which one line affects another, all of which are considered by PRIOSLICE. The probability computation can also be recursive because of control-flow and dependence-graph loops. As an example of multiple paths, consider line 1 in Figure 1 which defines all five input variables. Line 1 affects line 16 through its dependence successors, lines $3,6,9$, and 12, which control the calls to print Sqrt. These calls compute the parameter received by line 15 and used at line 16. (Line 1 also affects the inlined computations at the call sites in lines $4,7,10$, and 11.) PRIOSLICE takes into account all those effects, direct or indirect, of a line on any another line.

The dependence paths for some of those effects can overlap. Thus, those paths might not be probabilistically independent. However, it is generally impractical to compute the degree of probabilistic dependence among paths. For that reason, PRIOSLICE uses Fréchet inequalities [33] to obtain probability bounds of disjunctions of events whose dependence is unknown. The lower bound is the minimum of all probabilities and the upper bound is the sum of the probabilities or 1 if the sum is greater than 1. PRIOSLICE chooses the middle value of the lower and upper bounds as the probability of such a disjunction. For example, if the probabilities of line 1 affecting line 16 via the calls at lines $4,7,10,11$, and 13 are all 0.5 , the overall probability of line 1 affecting 16 is $(.5+\min (.5+.5+.5+.5+.5,1)) / 2=.75$.

After computing the probabilities for all dependencies, PRIOSLICE performs a backward best-first traversal ${ }^{3}$ [25] of the dependence graph from the slicing

${ }^{3}$ This can be seen as generalized breadth-first search where nodes to visit are picked by priority first, even if they are deeper in the graph. 
criterion using these probabilities. To illustrate, Table 1 shows the traversal steps from line 16 in our example. The first column shows the node-visit order and the second column lists the candidate nodes (discovered but not yet visited) and their affecting probabilities. In each step, PRIOSLICE picks the candidate (or set of candidates, if tied at the top) with the largest probability of affecting the failure and adds it (or them) to the prioritized slice (visited list) in the next row. When visiting a node, its undiscovered predecessors are added to the candidate list for the next step. For this example, if we simply assume that all branch probabilities are 0.5, PRIOSLICE traverses eight nodes to locate the fault at node 9 (as the table shows).

To compare PRIOSLICE with Weiser's static slicing and with thin slicing in this example, we measure how these other techniques localize the fault at line 9. Weiser's approach performs a breadth-first traversal from statement 16 . In this traversal, statement 16 is at depth 1 and the fault (statement 9) is at depth 5. There are ten reachable nodes before reaching depth 5 and there are three nodes at depth 5. If the goal is tied with other statements, we use the average of the best and worst cases to measure the effort of reaching that goal. Thus, the effort of locating the fault is $(11+13) / 2=12$, which is greater than the eight nodes needed by PRIOSLICE. Meanwhile, thin slicing does not discover the fault because all dependencies from line 9 are control dependencies. (If control dependencies are then considered, the fault is found for an even greater effort than Weiser's approach.)

PRIOSLICE uses points-to sets to model the probability that the memory location at a definition and use is the same. This is a common situation in most programs. In our example, however, there are no references or pointers, so these probabilities are all 1.

To study the cost-benefits of design choices for PRIOSLICE, we developed three variants called PRIOSLICE lite $_{\text {, PRIOSLICE }}$ succ, and PRIOSLICE update. These variants differ in two key aspects. First, when calculating the priority of a statement, one decision is whether to take all successors into account or those already visited. Second, during the traversal of the dependence graph, another decision is whether the priority of the statement gets updated while the statement is still in the priority queue. Table 2 summarizes the three variants of PRIOSLICE. Technically

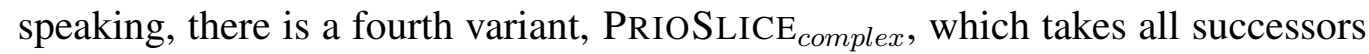
into account for calculating the priority of a statement and also continues updating the priorities of statements already discovered during the traversal. However, because of the high costs of this variant, we discarded it early in our study. 
Table 2: PRIOSLICE variants

\begin{tabular}{|l|c|c|}
\hline variant & all successors & priority updates \\
\hline PRIOSLICE $_{\text {lite }}$ & No & No \\
\hline PRIOSLICE $_{\text {succ }}$ & Yes & No \\
\hline PRIOSLICE $_{\text {update }}$ & No & Yes \\
\hline PRIOSLICE $\left._{\text {complex }}\right)$ & (Yes $)$ & (Yes) \\
\hline
\end{tabular}

\subsection{Probabilistic Model}

\subsubsection{Single Dependencies}

In addition to data dependencies, we also need to model control dependencies as a building block of PRIOSLICE. Here, we expand the propagation probability of data dependencies presented in Section 3.2 to both types of dependencies. These probabilities are necessary because any aspect of the program state (e.g., a fault) can propagate only through a sequence of dependencies of any type (data, control, or both). To obtain the propagation probability of a dependence, PRIOSLICE estimates how likely it is to cover (execute) the dependence such that an effect at the source of the dependence propagates to the target of that dependence.

The following notation describes the propagation probability of a dependence, assuming that its source has already been reached:

$$
P\left(a \stackrel{D}{\rightarrow}_{p} b\right)=\left\{\begin{array}{l}
P\left(a \stackrel{d d}{\rightarrow}_{p} b\right) \text { if } D \text { is a data dependence; } \\
P\left(a \stackrel{c d}{\rightarrow}_{p} b\right) \text { if } D \text { is a control dependence }
\end{array}\right.
$$

where $a \stackrel{D}{\rightarrow}_{p} b$ indicates that $(a, b)$ is a dependence of statement $b$ on $a$ and that a propagation occurs through this dependence. The marks $d d$ and $c d$ replace $D$ to specify data and control dependence, respectively. $P(a \stackrel{d d}{\rightarrow} b)$ was defined in Section 3.2.

A control dependence $(a, b)$ propagates an effect if and only if it is covered and an effect is transfered from the source to the target statement. For this type of dependence, the coverage probability is that of covering the corresponding controlflow edge $e$ (e.g., branch) and the transfer probability is the probability that $e$ is the wrong edge to take (i.e., the probability that, without the fault, a different successor of $a$ would have been taken). Thus, the propagation probability of a control dependence $(a, b)$ is 


$$
P\left(a \stackrel{c d}{\rightarrow}_{p} b\right)=P\left(a \rightarrow_{S} b\right) \times\left(1-P\left(a \rightarrow_{S} b\right)\right)
$$

\subsubsection{Dependence Graph}

PRIOSLICE prioritizes the inspection of statements by traversing the static dependence graph backward from the slicing criterion $C$ using a best-first search [25] based on the priority of each dependence. After visiting a node, its predecessors are discovered, the priorities of discovered nodes are calculated (or re-calculated if already discovered), and they are added to a priority queue. The node that is popped from the queue and visited next is the one with the highest priority in that queue.

For the traversal, PRIOSLICE weighs each dependence using two factors: its propagation probability (Section 4.3.1), which assumes that its source node has been reached, and the probability of reaching that source node from the entry of the program. The second factor is necessary in our model during the traversal because, of all dependencies with high propagation probabilities that can be visited next, those most likely to be reached at runtime are also the most likely to be the carriers of an effect that reaches $C$.

The probability $P_{\text {prio }}$ that PRIOSLICE assigns to a dependence $(a, b)$ during traversal is also conditioned to $b$ being already reached from the entry because, when the traversal visits $b$, it has already taken into account the probability that $b$ is the cause of the effect that reaches $C$. Therefore, the probability assigned by PRIOSLICE to a dependence $(a, b)$ during traversal is:

$$
\begin{aligned}
P_{\text {prio }}\left(a \stackrel{D}{\rightarrow}_{p} b\right) & =P\left(E N \rightarrow_{R} a \wedge a \stackrel{D}{\rightarrow}_{p} b \mid E N \rightarrow_{R} b\right) \\
& =\frac{P\left(E N \rightarrow_{R} a \wedge a \stackrel{D}{\rightarrow}_{p} b \wedge E N \rightarrow_{R} b\right)}{P\left(E N \rightarrow_{R} b\right)} \\
& =\frac{P\left(E N \rightarrow_{R} a\right) \times P\left(a \stackrel{D}{\rightarrow}_{p} b\right)}{P\left(E N \rightarrow_{R} b\right)}
\end{aligned}
$$

where $E N$ is the entry point of the program and $E N \rightarrow_{R} a$ (control-flow reachability defined in Section 3.1) and $a \stackrel{D}{\rightarrow}_{p} b$ are independent from each other. Also, these two events imply $E N \rightarrow_{R} b$. Thus, the traversal priority of a dependence is the product of the probabilities of reaching its source and propagating an effect to its target divided by the probability that the target has been reached.

To calculate or update the probability $P\left(a \rightarrow_{p} C\right)$ that a statement $a$ propagates an effect to $C$ through any sequence of dependencies, during the traversal with the 
information available so far, PRIOSLICE goes through all dependencies $(a, s)$ such that $s$ has been already visited or discovered using this formula:

$$
P\left(a \rightarrow_{p} C\right)= \begin{cases}1 & \text { if } a=C ; \\ P\left(\bigvee_{\substack{s \in \\ \operatorname{succ}_{T}(a)}} a \stackrel{s}{\rightarrow}_{p} C\right) & \text { otherwise }\end{cases}
$$

where $a \stackrel{s}{\rightarrow} C$ is the event that $a$ affects $C$ via dependence successor $s$ and where $\operatorname{succ}_{T}(a)$ depends on the variant of PRIOSLICE $T$ shown in Table 2. For PRIOSLICE $_{\text {lite }}$ and PRIOSLICE update, $\operatorname{succ}_{T}(a)$ is the subset of dependence successors of $a$ that have been visited (i.e., discovered nodes already pulled out of the priority queue). For PRIOSLICE succ $_{\text {, }} \operatorname{succ}_{T}(a)$ consists of all discovered nodes (i.e., either visited or still in the queue), which provides more data but at a greater cost.

For a dependence $(a, b)$, if $a \neq C, a$ affects $C$ via $b$ if and only if $a$ is reached at runtime, $a$ affects $t$ via this dependence, and $b$ affects $C$. Therefore, the probability that $a$ has an effect on the slicing criterion through a dependence successor $b$ is

$$
P\left(a \stackrel{b}{\rightarrow}_{p} C\right)=P_{\text {prio }}\left(a \stackrel{D}{\rightarrow}_{p} b\right) \times P\left(b \rightarrow_{p} C\right)
$$

which makes Equation 10 potentially recursive.

The terms in the disjunction in Equation 10, however, are not necessarily exclusive. One path from $a$ to $C$ might be probabilistically dependent on path from $a$ to $C$. However, it is impractical to compute the exact dependence relationships among those paths. Therefore, we estimate the disjunction of events whose dependence is unknown using Fréchet inequalities [33]:

$$
\max \left(P\left(A_{i}\right)\right) \leq P\left(\bigvee_{i=1}^{n} A_{i}\right) \leq \min \left(1, \sum_{i=1}^{n} A_{i}\right)
$$

Fréchet inequalities give the lower bound and upper bound of the probability of a disjunction of events. Without assuming any dependence among events, for simplicity, we estimate the probability as the middle point of the lower and upper bounds: 


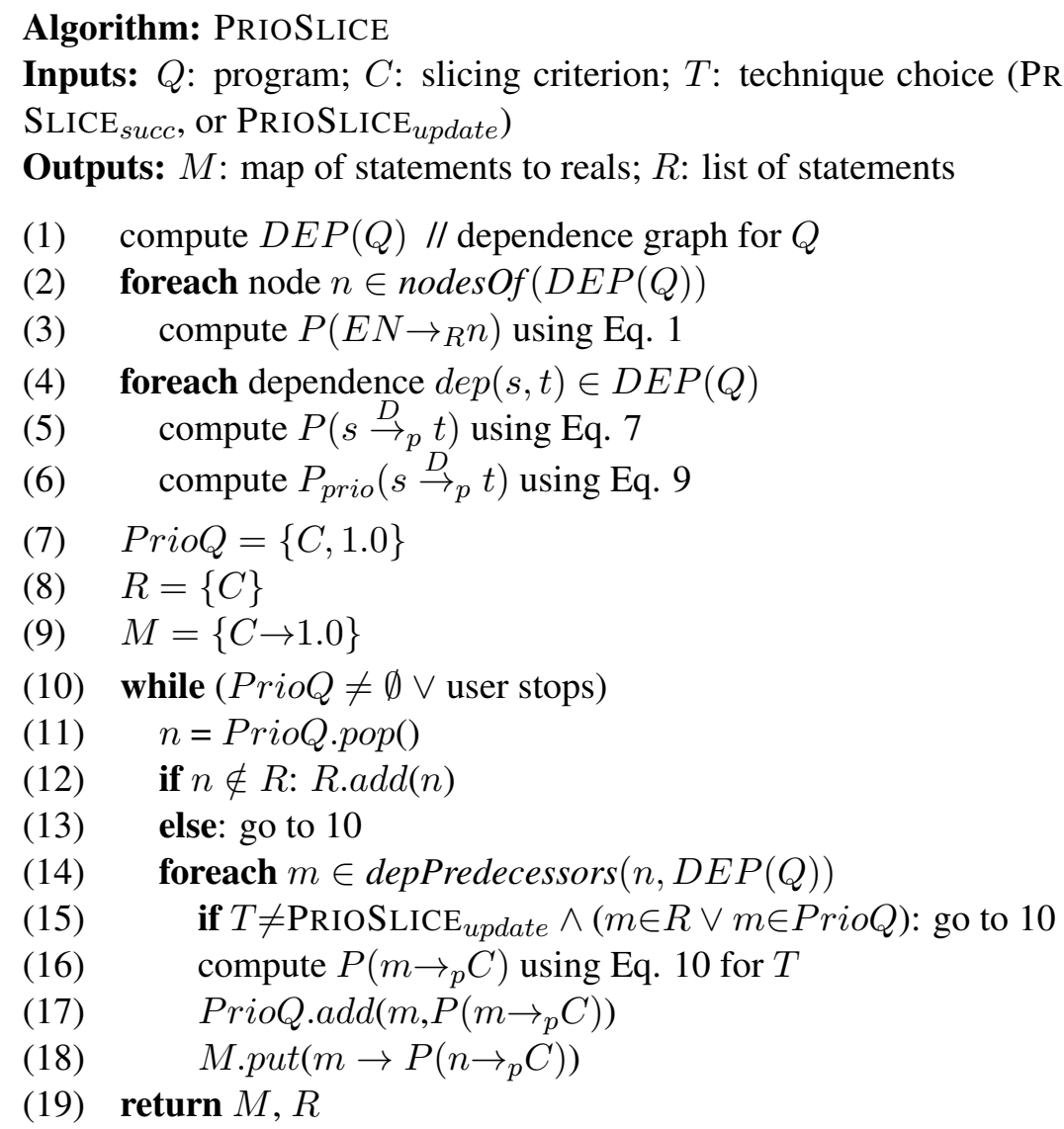

Outputs: $M$ : map of statements to reals; $R$ : list of statements

(1) compute $\operatorname{DEP}(Q) / /$ dependence graph for $Q$

(2) foreach node $n \in$ nodes $O f(D E P(Q))$

(3) compute $P\left(E N \rightarrow_{R} n\right)$ using Eq. 1

(4) foreach dependence $\operatorname{dep}(s, t) \in D E P(Q)$

(5) compute $P(s \stackrel{D}{\rightarrow} p t)$ using Eq. 7

(6) compute $P_{\text {prio }}\left(s \stackrel{D}{\rightarrow}_{p} t\right)$ using Eq. 9

(7) $\operatorname{Prio} Q=\{C, 1.0\}$

(8) $R=\{C\}$

(9) $M=\{C \rightarrow 1.0\}$

(10) while $($ Prio $Q \neq \emptyset \vee$ user stops $)$

(11) $n=$ PrioQ.pop()

(12) if $n \notin R: R . \operatorname{add}(n)$

(13) else: go to 10

(14) foreach $m \in$ depPredecessors $(n, D E P(Q))$

(15) if $T \neq$ PRIOSLICE $_{\text {update }} \wedge(m \in R \vee m \in$ Prio $Q)$ : go to 10

(16) compute $P\left(m \rightarrow{ }_{p} C\right)$ using Eq. 10 for $T$

(17) PrioQ.add $\left(m, P\left(m \rightarrow{ }_{p} C\right)\right)$

(18) $\quad M . p u t\left(m \rightarrow P\left(n \rightarrow{ }_{p} C\right)\right)$

(19) return $M, R$

Figure 5: Algorithm for prioritizing the traversal of dependence graphs

$$
P\left(\bigvee_{i=1}^{n} A_{i}\right)=\frac{\max \left(P\left(A_{i}\right)\right)+\min \left(1, \sum_{i=1}^{n} A_{i}\right)}{2}
$$

\subsection{Algorithm}

Figure 5 shows the PRIOSLICE algorithm that traverses the dependence graph of a program backward from a slicing criterion using a best-first strategy. The algorithm uses the equations presented in Sections 4.3 and 3 and takes the three 
variants of PRIOSLICE into account, according to the parameter $T$, via lines 15 and 16.

Line 1 computes the static dependence graph of the program from the slicing criterion. Lines 2-6 compute two sets of probabilities using our equations: the reaching probability from the program entry for each node in the graph and the conditional propagation probability from the entry of the program for each dependence. Lines 7-9 initialize the data structures for the priority queue and for the outputs, which are the probability map $M$ and the ranking list $R$, by seeding them with the slicing criterion and probability 1.

The main loop in lines 10-18 proceeds until no new nodes are left to process in the priority queue-until the graph has been completely traversed-or the user stops when a goal is found (e.g., a fault). Line 11 picks the next node $n$ to visit, which is the node with the highest priority, and removes it from the priority queue. ${ }^{4}$ Line 12 then appends $n$ to the ranking list if not already there. The visit of $n$ continues at lines 14-18 which, if the technique version $T$ allows (line 15), compute the propagation probabilities of the predecessors of $n$ in the graph and add (or update, according to $T$ ) those predecessors and their probabilities to the priority queue and the output map.

\section{Evaluation: Fault Localization}

To understand the effectiveness of PRIOSLICE in comparison with existing static-slicing approaches, we chose fault localization as the study application and performed an empirical evaluation on 35 faults across five real-world programs. Our goal was to determine how much of a program must be inspected by PRIOSLICE and two static-slicing approaches: Weiser's traversal approach and thin slicing [16]. Our research questions were:

RQ1: How effective is PRIOSLICE for fault localization compared to other static-slicing techniques?

RQ2: How do the variants of PRIOSLICE compare to each other?

RQ3: How effective is PRIOSLICE per fault localization effort?

RQ4: How practical is PRIOSLICE in terms of running costs?

The effectiveness of a fault-localization technique is inversely proportional to the effort a user needs to locate a fault using the corresponding dependence-graph

\footnotetext{
${ }^{4}$ Nodes tied with the highest priority are picked in the order they were inserted, which depends on how depPredecessors orders neighbors in the graph.
} 
visit order. The practicality is inversely proportional to the computational costs of the technique.

Unlike statistical fault localization, which provides seemingly-random orders to inspect code [26], static-slicing approaches such as PRIOSLICE navigate the dependence graph in order by visiting only unvisited neighbors - as suggested by Weiser [1]. Each new statement to visit is reached via an edge to another statement already visited. Navigating dependencies is a more natural way for developers to inspect programs [26].

\subsection{Experimental Setup}

We implemented PRIOSLICE in Java as a new module of DUA-FORENSICS, our dependence analysis and instrumentation toolset [27, 28]. Our implementation for this study inputs a Java-bytecode program, a failure location, and a fault to locate. It computes the static dependence graph from the failure point (slicing criterion), determines the traversal orders for PRIOSLICE, static slicing, and thin slicing, and determines the effort for locating the fault using each order. For simplicity, we assigned to all control-flow edges from the same statement the same probability (e.g., 0.5 for if-else branches). Our results show that this assumption was sufficient to demonstrate the effectiveness of PRIOSLICE.

Table 3 presents our subjects, their size in lines of code (LOC), and the number of faults studied per subject. NanoXML, XML-Security, JMeter, and Ant are provided by the SIR repository [34] along with faults seeded by other researchers and test suites that expose these faults, which we used to identify the failing points. Jaba is a Java-bytecode program-analysis framework provided upon request [35], including real faults found by its developers. PDFBox is provided by the Apache PDFBox SVN repository. To obtain the faults for PDFBox (and extra faults for other subjects as well), we used a random number generator to choose the fault locations and, for each location, we randomly applied one of the sufficient mutant operators identified by Offutt and colleagues [36].

Our criterion for selecting a fault for our study was that the fault is covered by at least one test case that exposes it by means of an unhandled exception or a different output. Table 4 lists the faults, as provided with those subjects, that satisfied our criterion for the study. Each fault is notated as $v X s Y$ or $v X m Y$. $v X$ stands for the version of the subject as it is in the repository. $s Y$ indicates that the seeded fault came along with the subject, where $Y$ conforms to the index of the fault in the repository. $m Y$ means that the fault was created by applying mutation to the subject, and the index $Y$ simply followed a natural increasing order of integers from one. For NanoXML, XML-Security, JMeter, and Jaba, 
Table 3: Subjects and their characteristics

\begin{tabular}{|l||r|r|r|}
\hline subject & description & LOC & faults \\
\hline NanoXML & XML parser & 3521 & 10 \\
\hline XML-Security & encryption library & 22361 & 10 \\
\hline JMeter & performance tester & 35547 & 10 \\
\hline Jaba & program analyzer & 37920 & 10 \\
\hline Ant & project builder & 57867 & 10 \\
\hline PDFBox & PDF library & 59610 & 10 \\
\hline
\end{tabular}

Table 4: Identifiers of the faults used per subject

\begin{tabular}{|l|r|}
\hline subject & fault ids \\
\hline NanoXML & $\mathrm{v} 1 \mathrm{~s} 1, \mathrm{v} 1 \mathrm{~s} 2, \mathrm{v} 1 \mathrm{~s} 3, \mathrm{v} 1 \mathrm{~s} 4, \mathrm{v} 1 \mathrm{~s} 5, \mathrm{v} 1 \mathrm{~s} 6, \mathrm{v} 1 \mathrm{~s} 7, \mathrm{v} 1 \mathrm{~m} 1, \mathrm{v} 1 \mathrm{~m} 2, \mathrm{v} 1 \mathrm{~m} 3$ \\
\hline XML-security & $\mathrm{v} 1 \mathrm{~s} 2, \mathrm{v} 1 \mathrm{~s} 3, \mathrm{v} 1 \mathrm{~s} 5, \mathrm{v} 1 \mathrm{~s} 14, \mathrm{v} 1 \mathrm{~s} 16, \mathrm{v} 1 \mathrm{~s} 17, \mathrm{v} 1 \mathrm{~s} 20, \mathrm{v} 1 \mathrm{~m} 1, \mathrm{v} 1 \mathrm{~m} 2, \mathrm{v} 1 \mathrm{~m} 3$ \\
\hline JMeter & $\mathrm{v} 2 \mathrm{~s} 1, \mathrm{v} 2 \mathrm{~s} 2, \mathrm{v} 2 \mathrm{~s} 5, \mathrm{v} 2 \mathrm{~s} 6, \mathrm{v} 2 \mathrm{~s} 7, \mathrm{v} 2 \mathrm{~s} 11, \mathrm{v} 2 \mathrm{~s} 19, \mathrm{v} 2 \mathrm{~m} 1, \mathrm{v} 2 \mathrm{~m} 2, \mathrm{v} 2 \mathrm{~m} 3$ \\
\hline Jaba & $\mathrm{v} 1 \mathrm{~s} 2, \mathrm{v} 1 \mathrm{~s} 5, \mathrm{v} 1 \mathrm{~s} 7, \mathrm{v} 1 \mathrm{~s} 11, \mathrm{v} 1 \mathrm{~s} 12, \mathrm{v} 1 \mathrm{~s} 13, \mathrm{v} 1 \mathrm{~s} 17, \mathrm{v} 1 \mathrm{~m} 1, \mathrm{v} 1 \mathrm{~m} 2, \mathrm{v} 1 \mathrm{~m} 3$ \\
\hline Ant & $\mathrm{v} 2 \mathrm{~s} 3, \mathrm{v} 2 \mathrm{~s} 6, \mathrm{v} 2 \mathrm{~s} 7, \mathrm{v} 2 \mathrm{~s} 8, \mathrm{v} 2 \mathrm{~m} 1, \mathrm{v} 2 \mathrm{~m} 2, \mathrm{v} 2 \mathrm{~m} 3, \mathrm{v} 2 \mathrm{~m} 4 \mathrm{v} 2 \mathrm{~m} 5, \mathrm{v} 2 \mathrm{~m} 6$ \\
\hline PDFBox & $\mathrm{v} 0 \mathrm{~m} 1, \mathrm{v} 0 \mathrm{~m} 2, \mathrm{v} 0 \mathrm{~m} 3, \mathrm{v} 0 \mathrm{~m} 4, \mathrm{v} 0 \mathrm{~m} 5, \mathrm{v} 0 \mathrm{~m} 6, \mathrm{v} 0 \mathrm{~m} 7, \mathrm{v} 0 \mathrm{~m} 8, \mathrm{v} 0 \mathrm{~m} 9, \mathrm{v} 0 \mathrm{~m} 10$ \\
\hline
\end{tabular}

exactly seven faults provided with the subject satisfied our criterion. For Ant, four faults satisfied. In order to have ten faults for each subject for consistency, we seeded the rest of the faults (three for NanoXML, XML-Security, JMeter and Jaba, six for Ant, and ten for PDFBox), documented in our PRIOSLICE website ${ }^{5}$ , using the aforementioned mutation approach.

We performed all our experiments in a quad-core Intel machine with 48 GB RAM running 64-bit Linux and Oracle/Sun's JVM 7.

\subsection{Methodology}

Our experimental process used the following steps for each fault:

1. Identify the failing point. To locate the failure, we took the first test case for the subject that fails for the given fault and identified the first statement involved in that failure. The failing statement is either the one that prints the first wrong value, the statement that throws an uncaught exception, or the conditional statement that skips the printing of the first missing output value. This

\footnotetext{
${ }^{5}$ http://nd.edu/ yzhang20/priosli
} 
step reflects the process of Figure 3 where developers to identify the failure reported by users. Our website for PRIOSLICE ${ }^{5}$ documents each failing point we identified.

2. Obtain the static dependence graph. We applied DUA-FORENSICS with this failing point as the slicing criterion to obtain the portion of the static dependence graph that affects that point. This process corresponds to the first step in Figure 4.

3. Apply each technique. We applied each traversal technique-PRIOSLICE, Weiser's approach, and thin slicing - and measured the respective efforts, as fractions of the program, required to reach (localize) the first faulty statement $f$ using this formula:

$$
\text { effort }(f)=\frac{\operatorname{rank}(f)}{\text { program size }}
$$

where $\operatorname{rank}(f)$ is the number of statements that had to be visited to reach $f$ and program size is the total number of statements in the program. In case of a tie, when the visit order of $f$ is the same as for other statements, we defined $\operatorname{rank}(n)$ as the average visit order of $f$ and all statements tied with it. For example, if four statements are tied at positions 3-6 (i.e., visited together after the first two visited nodes), their rank is $(3+6) / 2=4.5$.

For Weiser's approach and for thin slicing, we used breadth-first backward traversals of the graphs to determine the order in which the statements in the slices are inspected. For thin slicing, we omitted control dependencies and basevariable data dependencies (i.e. dependencies where the variable at the use is a reference to an object for which a field is accessed or a method is called) [16]. We implemented thin slicing without the iterative addition of initially-omitted dependencies mentioned by its authors because there is no clear order in which those dependencies are added next [16].

\subsection{Results and Analysis}

Tables 5 and 6 show the average fault-localization effort per subject and overall for the three variants of PRIOSLICE, Weiser's approach (WSlice), and thin slicing. Thin slicing was applicable to only twelve faults. For the other 48 faults, it did not find the fault. Therefore, Table 6 shows average results only for those twelve faults. The best result in each row of each table is shown in bold.

To illustrate, in these tables, an effort of $10 \%$ indicates that the faulty statement is found by traversing $10 \%$ of the dependence graph of the program. In Table 6 , 
Table 5: Average localization effort per subject and overall of using each variant of PRIOSLICE and Weiser's approach (WSlice)

\begin{tabular}{|l|r|r|r|r|r|}
\hline \multicolumn{1}{|c|}{ Subjects } & Applicable faults & PRIOSLICE $_{\text {lite }}$ & PRIOSLICE $_{\text {succ }}$ & PRIOSLICE $_{\text {update }}$ & WSlice \\
\hline NanoXML & 10 & $19.22 \%$ & $19.29 \%$ & $\mathbf{1 5 . 2 0 \%}$ & $17.36 \%$ \\
\hline XML-Security & 10 & $12.50 \%$ & $\mathbf{1 0 . 5 1 \%}$ & $13.63 \%$ & $22.59 \%$ \\
\hline JMeter & 10 & $\mathbf{9 . 8 9 \%}$ & $12.64 \%$ & $16.34 \%$ & $20.11 \%$ \\
\hline Jaba & 10 & $\mathbf{1 7 . 1 6 \%}$ & $17.63 \%$ & $22.52 \%$ & $30.50 \%$ \\
\hline Ant & 10 & $7.48 \%$ & $\mathbf{3 . 2 8} \%$ & $5.88 \%$ & $34.28 \%$ \\
\hline PDFBox & 10 & $14.70 \%$ & $13.95 \%$ & $\mathbf{1 0 . 5 2 \%}$ & $34.66 \%$ \\
\hline \multicolumn{2}{|r|}{ Average all applicable faults: } & $13.49 \%$ & $\mathbf{1 2 . 8 8 \%}$ & $14.01 \%$ & $26.58 \%$ \\
\hline
\end{tabular}

Table 6: Average localization effort per subject and overall of using each variant of PRIOSLICE, thin slicing, and Weiser's approach (WSlice)

\begin{tabular}{|c|c|c|c|c|c|c|}
\hline Subjects & Applicable faults & PRIOSLICE $_{\text {lite }}$ & PriOSliCE $_{\text {succ }}$ & PRIOSLICE $_{\text {update }}$ & Thin Slice & WSlice \\
\hline NanoXML & 4 & $25.03 \%$ & $25.20 \%$ & $19.78 \%$ & $27.23 \%$ & $27.95 \%$ \\
\hline XML-Security & 0 & not applicable & not applicable & not applicable & not applicable & not applicable \\
\hline JMeter & 2 & $16.60 \%$ & $10.96 \%$ & $17.28 \%$ & $1.89 \%$ & $4.05 \%$ \\
\hline Jaba & 3 & $18.77 \%$ & $20.92 \%$ & $24.58 \%$ & $17.38 \%$ & $32.96 \%$ \\
\hline Ant & 1 & $3.99 \%$ & $6.78 \%$ & $14.64 \%$ & $28.76 \%$ & $44.04 \%$ \\
\hline PDFBox & 2 & $4.56 \%$ & $7.08 \%$ & $7.77 \%$ & $13.93 \%$ & $13.01 \%$ \\
\hline \multicolumn{2}{|c|}{ Average all applicable faults: } & $16.89 \%$ & $17.20 \%$ & $18.13 \%$ & $18.46 \%$ & $24.07 \%$ \\
\hline
\end{tabular}




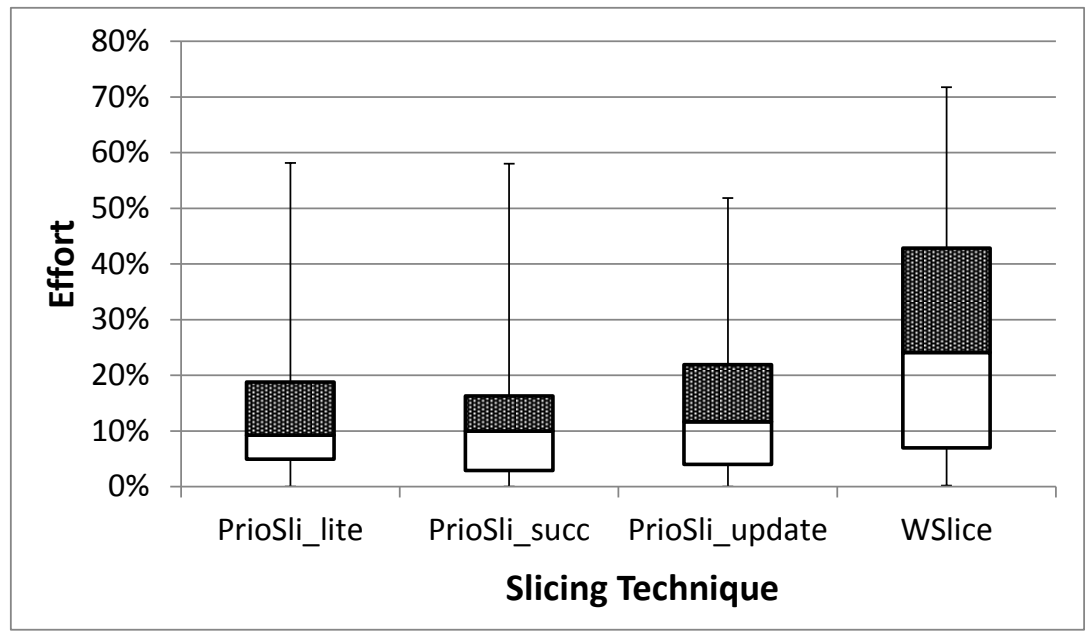

Figure 6: Distribution of localization efforts for all subjects

not applicable indicates that the techniques cannot be compared because thin slicing was not applicable to any faults for the corresponding row (i.e., each of those faults can be reached through at least one control dependence or base-variable data dependence).

We also analyzed the distribution and statistical significance of the results. First, we used the Wilcoxon signed-rank one-tailed test [37], a non-parametric test that makes no assumptions about the data distribution, to assess whether our technique is more effective than static and thin slicing. We chose 0.05 as the significance level. Tables 7 and 8 show the statistical test results. Because of the small differences between for PRIOSLICE and thin slicing when thin slicing is applicable, we conducted both directional and non-directional tests for Table 8 . In those tables, success indicates that Treatment 1 (the PRIOSLICE variant) is significantly better than Treatment 2 (Weiser's approach or thin slicing, respectively).

Table 7: Wilcoxon signed-rank test results comparing PRIOSLICE variants with Weiser's approach (WSlice) for significance level .05

\begin{tabular}{|l|l|r|r|r|r|}
\hline \multirow{2}{*}{ Treatment 1 } & \multirow{2}{*}{ Treatment 2 } & \multirow{2}{*}{ Sample size } & \multicolumn{4}{|c|}{ Directional test } \\
\cline { 4 - 7 } & & Z-value & Critical Z & Result \\
\hline PRIOSLICE $_{\text {lite }}$ & WSlice & 60 & -3.6808 & 1.645 & success \\
\hline PRIOSLICE $_{\text {succ }}$ & WSlice & 60 & -3.5777 & 1.645 & success \\
\hline PRIOSLICE $_{\text {update }}$ & WSlice & 60 & -3.5998 & 1.645 & success \\
\hline
\end{tabular}




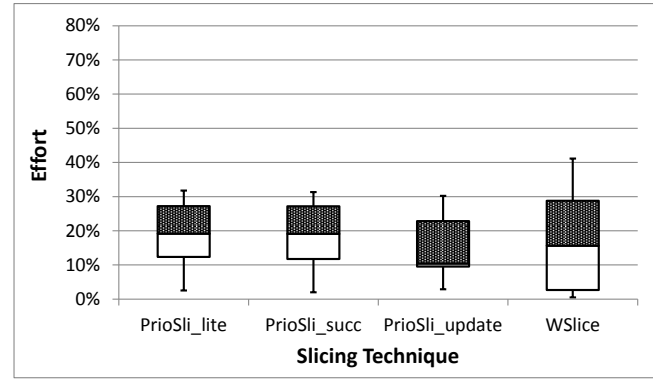

(a) Boxplot for NanoXML

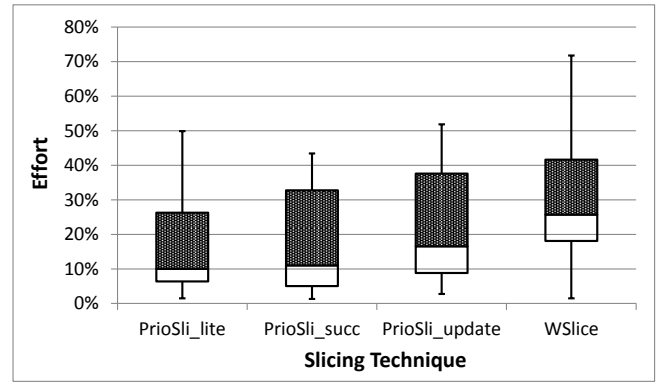

(c) Boxplot for JMeter

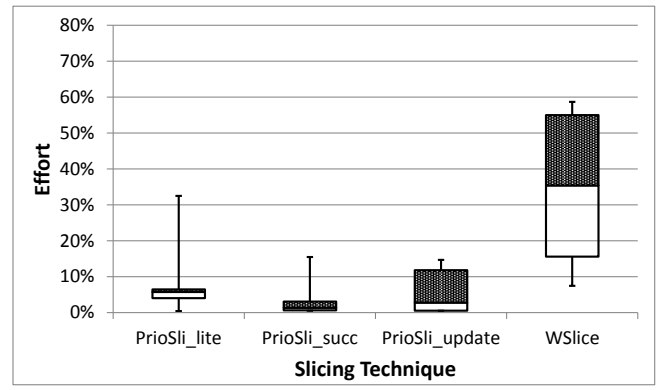

(e) Boxplot for Ant

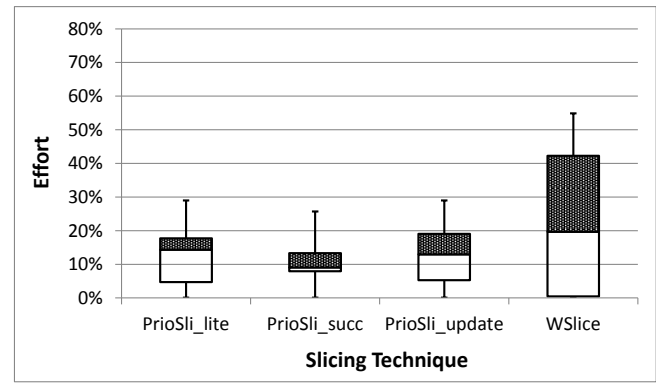

(b) Boxplot for XML-Security

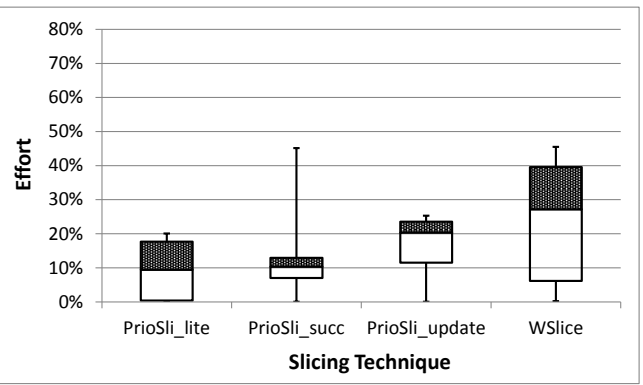

(d) Boxplot for Jaba

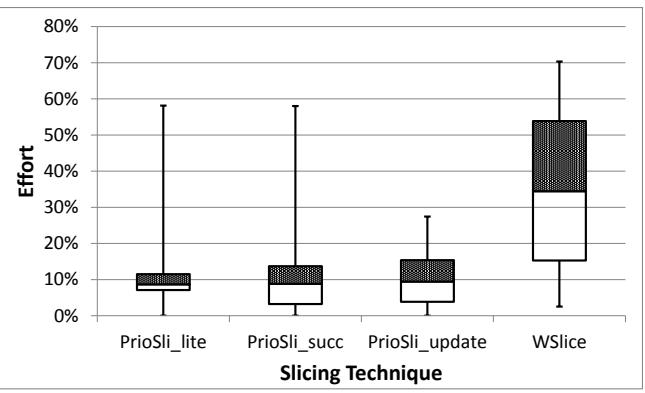

(f) Boxplot for PDFBox

Figure 7: Distribution of localization efforts for individual subjects 


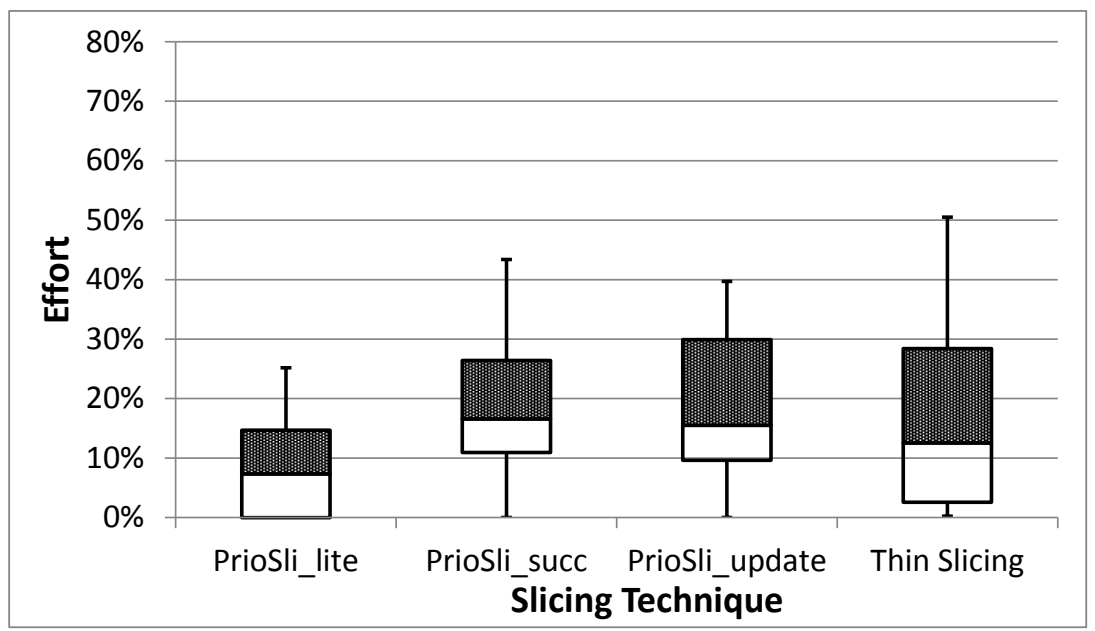

Figure 8: Distribution of localization efforts for the twelve faults that are applicable to thin slicing

Table 8: Wilcoxon signed-rank test results comparing PRIOSLICE variants with thin slicing for significance level .05

\begin{tabular}{|l|l|r|r|r|r|r|r|r|}
\hline \multirow{2}{*}{ Treatment 1 } & \multirow{2}{*}{ Treatment 2 } & \multirow{2}{*}{ Sample size } & \multicolumn{4}{|c|}{ Directional } & \multicolumn{3}{c|}{ Non-directional test } \\
\cline { 4 - 10 } & & & W-value & Critical W & Result & W-value & Critical W & Result \\
\hline PRIOSLICE $_{\text {lite }}$ & Thin Slice & 12 & 36 & 17 & fail & 36 & 13 & fail \\
\hline PRIOSLICE $_{\text {succ }}$ & Thin Slice & 12 & 36 & 17 & fail & 36 & 13 & fail \\
\hline PRIOSLICE $_{\text {update }}$ & Thin Slice & 12 & 38 & 17 & fail & 38 & 13 & fail \\
\hline
\end{tabular}

Second, we created boxplot diagrams for all faults in all subjects (Figure 6) and for each subject (Figure 7(a)-7(f)) to compare the effort distributions for the PRIOSLICE variants and Weiser's approach. We also created a boxplot diagram for the twelve faults that are applicable to thin slicing (Figure 8) to compare PRIOSLICE variants and thin slicing. The line inside each box represents the median effort, the box represents the second-to-third quartile range, and the whiskers show the lower and upper ends of the distributions. Figure 8 shows the boxplots for the faults for which thin slicing was applicable.

Finally, we studied the performance of each technique in fault localization. Table 9 shows the averages and standard deviations of the running times in seconds for all techniques per subject and for all subjects (all faults). For thin slicing, the times are included for all traversals, including those for which it did not find the faults. Table 10 and 11 present the time-cost effectiveness (TCE) of applying each technique per subject and overall. Time-cost effectiveness indicates the total time used to locate fault $f$ using technique tech (i.e. either of the three variants 
of PRIOSLICE, Weiser's slicing, or thin slicing), therefore a smaller number indicates better time-cost effectiveness. We calculated its value using formula below:

$$
T C E(\text { tech }, f)=T(\text { tech })+\operatorname{rank}(f) \times t
$$

where $T($ tech $)$ is the running time for the technique, $\operatorname{rank}(\mathrm{f})$ is the number of statements that had to be traversed to reach $f$ using emph, and $t$ is the average inspection time a person spends on one statement. The equation above implicitly includes a divisor 1 , because in this case the number of faults is simply one. We then obtained TCE for all subjects using all techniques by:

$$
T C E=\frac{\sum_{\text {tech }} \sum_{f} T C E(\text { tech }, f)}{N}
$$

where $N$ is the total number of faults. We did not simply divide running times in Table 9 by efforts in Table 5 and Table 6 to obtain time-cost effectiveness, because the running times are only for running the technique but not localizing faults. Instead, we add slice inspection time $t$ with running times of the technique to evaluate how much time is needed to localize each fault. For the inspection time, we set it as 15 seconds, 30 seconds, 1 minute, 10 minutes, 30 minutes, 1 hours, 4 hours, and 8 hours (standard working hours for 1 person per day), to study how it affects the effectiveness of PRIOSLICE and other static slicing techniques in a fault localization task. For data readability, we converted time from seconds into minutes.

\subsubsection{RQ1: Effectiveness of PRIOSLICE}

The results in Tables 5 indicate that the three variants of PRIOSLICE find the faults faster than Weiser's approach (WSlice) by more than $10 \%$ of the program on average for all subjects, which is a considerable difference. Also, the three variants outperform Weiser's approach for every subject except for NanoXML-

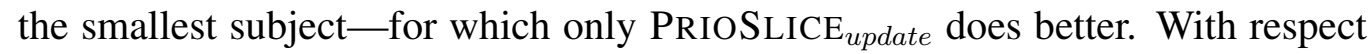
to thin slicing, PRIOSLICE found all 60 faults unlike thin slicing, which could only find twelve of them. But even for those faults that thin slicing could find, all three variants of PRIOSLICE required a lightly lower effort on average. For those eight faults, Weiser's approach was, again, uncompetitive.

The boxplots also show that Weiser's approach and thin slicing have a greater variance than PRIOSLICE. The main reason for this greater variance is that the distance between fault and failure in the dependence graph can be unpredictable, which affects breadth-first traversals more than focused traversals such as PRIOSLICE. When the fault is close to the failure, all techniques incur a small effort, 
which explains their similarity for the first quartiles. For the top quartiles, naturally, it still takes PRIOSLICE a greater-than-average effort, but this is much less than for the existing techniques.

We manually inspected specific cases in which Weiser's approach was more effective than PrIOSLICE. For example, for fault v1s1 in NanoXML, PRIOSLICE $_{\text {update }}$ traverses $24.4 \%$ of the program to locate the fault whereas Weiser's slicing only needs $4.7 \%$. The shortest dependence path between failure and fault has four dependencies, three of which are control dependencies. There are also many data-dependence dominated paths between non-faulty statements and the failing point. Therefore, in this particular case, and despite considering control dependencies, PRIOSLICE is lured away by more promising data dependencies while a simpler breadth-first search finds the fault faster.

With respect to thin slicing, PRIOSLICE was more effective for two reasons. First, PRIOSLICE guaranteed that all faults would be found, whereas thin slicing was not applicable in more than half of all cases. We confirmed that, in those cases that thin slicing could not locate the fault, the dependencies from the faulty code are control dependencies, which are discarded by thin slicing. (A user of thin slicing could add them later after exhausting all data dependencies, although the visit order of control dependencies is not specified and the localization effort would be quite large in the end.) Second, when thin slicing was applicable, PRIOSLICE was still better on average by discriminating among data dependencies and thus traverse slices faster towards the fault. However, in two cases (i.e. JMeter and Jaba), thin slicing followed data dependencies which seemed weak to PRIOSLICE but happened to propagate the fault.

Regarding statistical strength, for Weiser's approach, we used a non-parametric, directional (one-tailed) Wilcoxon signed-rank test [37] whereas, for thin slicing, we used both directional (one-tailed) and non-directional versions because of the small differences observed. Table 7 indicates that all variants of PRIOSLICE are significantly more effective than Weiser's approach because the absolute values of $\mathrm{Z}$ are greater than the critical values of $\mathrm{Z}$. Table 8 , which uses $\mathrm{W}$ values instead of $\mathrm{Z}$ because of the small sample sizes, shows that, for the cases in which thin slicing is applicable (twelve faults), we cannot conclude that PRIOSLICE is better or that the two techniques perform differently. (Of course, PRIOSLICE is better for the other 48 faults for which thin slicing was not applicable.)

Finally, from the distributions shown in Figures 6 and 7(a)-7(f), we make two main observations. First, the median effort of using Weiser's approach is larger than using each variant of PRIOSLICE overall and for most of the subjects individually. The exceptions are the medians of PRIOSLICE lite $_{\text {and PIOSLICE }}$ acc for 
the smaller subject, NanoXML. Second, Weiser's approach showed a greater variability in all cases, and therefore it was less predictable than PRIOSLICE. Hence, these distributions confirm that the efforts of using PRIOSLICE to locate faults are spread over a shorter range. Therefore, all PRIOSLICE variants were not only more effective on average but also more stable than Weiser's approach. From the distributions in Figure 8, we also observe that PRIOSLICE performs similarly to thin slicing when thin slicing is applicable but with a slightly lower variability.

\subsubsection{RQ2: Comparison of PRIOSLICE Variants}

To explain the differences among the variants of PRIOSLICE, let us recall the two factors that distinguish them. First, when calculating the priority of the statement, PRIOSLICE succ takes all successors into account but PRIOSLICE lite $_{\text {and }}$

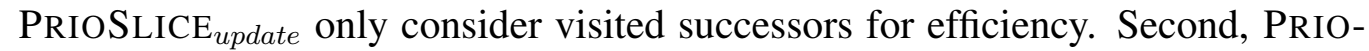
SLICE $_{\text {update }}$ continues updating the priorities of nodes after visiting them to propa-

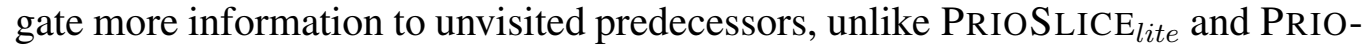
$\mathrm{SLICE}_{\text {succ }}$ for which the priorities in the priority do not change. Yet, it was not easy to predict beforehand how much each factor would affect the results.

The results for all faults in the last row of Table 5 show that these factors actually had a small but non-negligible influence. PRIOSLICE succ $_{\text {outperformed }}$ PRIOSLICE ${ }_{\text {lite }}$ by $0.61 \%$ and, somewhat surprisingly, also did better than PRIOSLICE $_{\text {update }}$ by $1.13 \%$. PRIOSLICE lite worked better for JMeter and Jaba, PRIOSLICE $_{\text {succ }}$ was the most effective variant for XML-Security and Ant, and PRIOSLICE $_{\text {update }}$ was better for NanoXML and PDFBox. These results suggest that the effects of each factor depend on the subject. Interestingly, the differences among variants were large for most subjects, despite almost canceling each other on average. These results per variant should also be put in perspective of the costs of using each variant, which we analyze for RQ4.

\subsubsection{RQ3: Effectiveness per Localization Effort}

Another important view of the benefits of fault-localization techniques corresponds to their cost-effectiveness because developers often want to know what to expect while inspecting the program-how much more effort they might need to spend to find a fault. In some cases, developers might even stop when reaching an inspection budget if the failure is not critical. In all such cases, it is useful to estimate how many faults a fault-localization technique finds per localization effort.

Figure 9 shows the effectiveness of PRIOSLICE and Weiser's approach per fault-localization effort. We normalized the inspection effort of each fault (hori- 


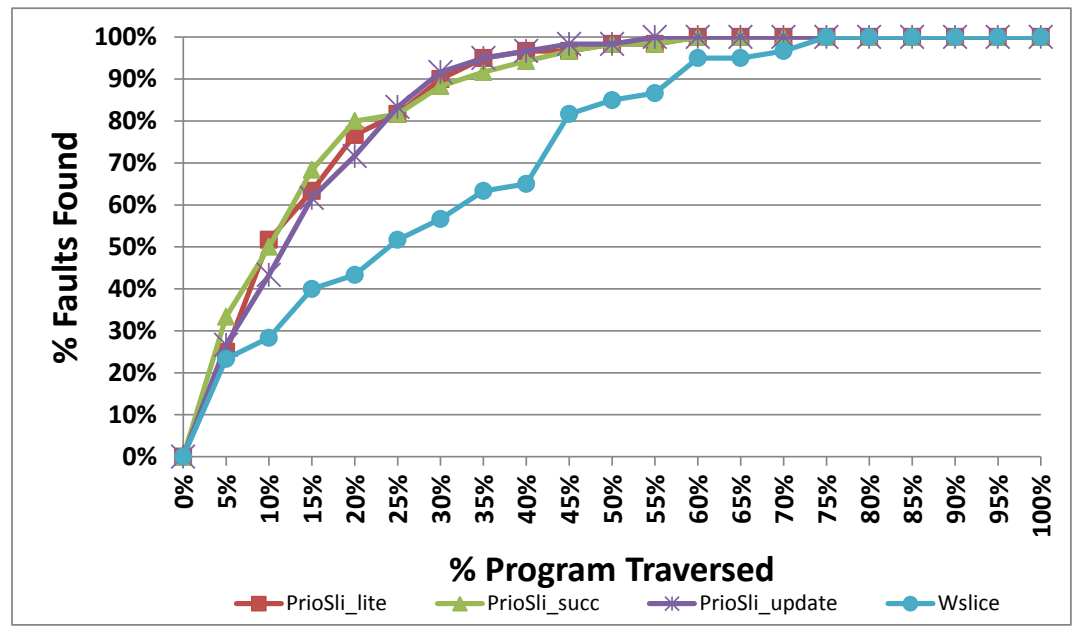

Figure 9: Effectiveness per localization effort

zontal axis) as a percentage of the subject it belongs to. The vertical axis represents the percentage of all faults (a total of 60 in this study) found.

The figure shows that PRIOSLICE and Weiser's slicing perform about the same for the first $5 \%$ of the program. Beyond that point, PRIOSLICE finds about $20 \%$ more of the faults than Weiser's approach for the first $10 \%$ of the program. Between $10 \%$ and $40 \%$ of the program, PRIOSLICE continues to outperform Weiser's approach by even greater margins. Only by $75 \%$ of the program Weiser's approach catches PRIOSLICE in localizing all 35 faults. Regarding the three vari-

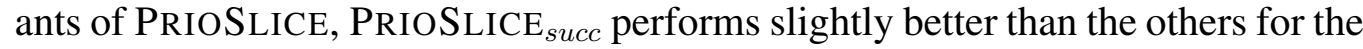
first $20 \%$.

In all, the results suggest that PRIOSLICE not only can find faults faster on average than Weiser's approach, but its superiority is even larger, comparatively, if the developer is working under a budget. Thus, PRIOSLICE can greatly benefit developers in finding more faults faster than existing approaches.

\subsubsection{RQ4: Practicality of PRIOSLICE}

Table 9 presents the average running times and its standard deviation for each technique per subject and for all subjects (all faults). For all subjects, our implementation of PRIOSLICE took longer than Weiser's approach (WSlice) and thin slicing as it performed static slicing before computing the probabilities.

For NanoXML, this difference was not important-about 4 extra seconds on average for PRIOSLICE 
Table 9: Statistics for running times of all techniques in seconds

\begin{tabular}{|l|r|r|r|r|r|r|}
\hline \multicolumn{2}{|c|}{ Subjects } & PrIOSLICE $_{\text {lite }}$ & PRIOSLICE $_{\text {succ }}$ & PRIOSLICE $_{\text {update }}$ & WSlice & Thin Slice \\
\hline \multirow{2}{*}{ NanoXML } & avg & $\mathbf{1 4 . 8}$ & $\mathbf{2 6 . 2}$ & $\mathbf{1 5 . 0}$ & $\mathbf{1 1 . 3}$ & $\mathbf{1 3 . 3}$ \\
\cline { 2 - 7 } & st.dev & 0.3 & 1.1 & 0.2 & 0.4 & 1.4 \\
\hline \multirow{3}{*}{ XML-Security } & $\mathbf{a v g}$ & $\mathbf{2 0 4 . 7}$ & $\mathbf{1 3 0 1 . 9}$ & $\mathbf{2 2 2 . 3}$ & $\mathbf{5 2 . 7}$ & $\mathbf{6 4 . 3}$ \\
\cline { 2 - 8 } & st.dev & 3.6 & 98.1 & 3.6 & 3.6 & 3.6 \\
\hline \multirow{3}{*}{ JMeter } & $\mathbf{a v g}$ & $\mathbf{8 9 0 . 1}$ & $\mathbf{3 7 7 7 . 6}$ & $\mathbf{9 3 3 . 5}$ & $\mathbf{1 7 5 . 2}$ & $\mathbf{1 8 2 . 5}$ \\
\cline { 2 - 8 } & st.dev & 76.1 & 72.8 & 66.4 & 23.3 & 31.4 \\
\hline \multirow{3}{*}{ Jaba } & $\mathbf{a v g}$ & $\mathbf{6 5 2 . 9}$ & $\mathbf{2 6 8 1 . 4}$ & $\mathbf{8 5 8 . 5}$ & $\mathbf{1 5 5 . 4}$ & $\mathbf{1 5 6 . 6}$ \\
\cline { 2 - 8 } & st.dev & 17.4 & 110.4 & 11.5 & 7.0 & 5.9 \\
\hline \multirow{3}{*}{ Ant } & $\mathbf{a v g}$ & $\mathbf{1 8 6 8 . 7}$ & $\mathbf{9 4 9 1 . 5}$ & $\mathbf{2 0 0 0 . 6}$ & $\mathbf{4 2 1 . 5}$ & $\mathbf{4 2 6 . 7}$ \\
\cline { 2 - 8 } & st.dev & 21.3 & 255.0 & 7.2 & 9.7 & 11.6 \\
\hline \multirow{2}{*}{ PDFBox } & $\mathbf{a v g}$ & $\mathbf{2 0 3 1 . 1}$ & $\mathbf{6 8 8 2 . 3}$ & $\mathbf{2 3 2 6 . 5}$ & $\mathbf{2 1 2 . 0}$ & $\mathbf{2 1 3 . 5}$ \\
\cline { 2 - 8 } & st.dev & 15.2 & 130.9 & 38.9 & 6.1 & 6.0 \\
\hline \multirow{2}{*}{ all faults } & $\mathbf{a v g}$ & $\mathbf{9 4 2 . 6}$ & $\mathbf{4 0 3 3 . 0}$ & $\mathbf{1 0 5 5 . 0}$ & $\mathbf{1 7 1 . 3}$ & $\mathbf{1 7 4 . 9}$ \\
\cline { 2 - 7 } & st.dev & 775.4 & 3282.4 & 859.8 & 133.4 & 134.6 \\
\hline
\end{tabular}

Table 10: Time-cost effectiveness for applying PRIOSLICE variants and Weiser's slicing (WSlice) on all faults

\begin{tabular}{|c|c|c|c|c|}
\hline subjects & PrIOSLICE $_{\text {lite }}$ & PrIOSLICE $_{\text {succ }}$ & PRIOSLICE $_{\text {update }}$ & WSlice \\
\hline NanoXML & 41.7 & 41.8 & 33.0 & 37.7 \\
\hline XML-Security & 168.5 & 143.6 & 183.8 & 255.9 \\
\hline JMeter & 205.7 & 267.2 & 338.7 & 415.2 \\
\hline Jaba & 452.8 & 468.6 & 594.1 & 803.0 \\
\hline Ant & 242.1 & 120.5 & 191.3 & 1095.7 \\
\hline PDFBox & 530.1 & 511.4 & 380.5 & 1240.1 \\
\hline average & 273.5 & 258.9 & 286.9 & 641.3 \\
\hline
\end{tabular}


Table 11: Time-cost effectiveness for applying PRIOSLICE variants, Weiser's slicing (WSlice) and thin slicing (Thin Slice) on the twelve faults that are applicable to thin slicing

\begin{tabular}{|l|r|r|r|r|r|}
\hline \multicolumn{1}{|c|}{ Subjects } & PrIOSLICE $_{\text {lite }}$ & PrIOSLICE $_{\text {succ }}$ & PrIOSLICE $_{\text {update }}$ & \multicolumn{1}{c|}{ WSlice } & \multicolumn{1}{c|}{ Thin Slice } \\
\hline NanoXML & 54.3 & 54.7 & $\mathbf{4 2 . 9}$ & 60.6 & 59.1 \\
\hline XML-Security & not applicable & not applicable & not applicable & not applicable & not applicable \\
\hline JMeter & 344.0 & 232.4 & 358.2 & 84.0 & $\mathbf{3 9 . 3}$ \\
\hline Jaba & 494.9 & 555.0 & 648.2 & 867.5 & $\mathbf{4 5 7 . 6}$ \\
\hline Ant & $\mathbf{1 3 0 . 6}$ & 232.3 & 471.0 & 1407.5 & 919.5 \\
\hline PDFBox & $\mathbf{1 6 7 . 0}$ & 265.5 & 282.4 & 466.8 & 497.7 \\
\hline average & $\mathbf{2 3 8 . 2}$ & 268.0 & 360.5 & 577.3 & 394.6 \\
\hline
\end{tabular}

Table 12: Average time-cost effectiveness for applying PRIOSLICE variants and Weiser's slicing (WSlice) on all faults for each inspection time $t$ a programmer spends on each statement in the slice

\begin{tabular}{|l|r|r|r|r|}
\hline & PrIOSLICE $_{\text {ite }}$ & PRIOSLICE $_{\text {succ }}$ & PRIOSLICE $_{\text {update }}$ & \multicolumn{1}{c|}{ WSlice } \\
\hline $\mathbf{t = 1 5 s}$ & $\mathbf{1 1 7 . 6}$ & 124.1 & 128.7 & 229.5 \\
\hline $\mathbf{t}=\mathbf{3 0 s}$ & 273.5 & $\mathbf{2 5 8 . 9}$ & 286.9 & 641.3 \\
\hline $\mathbf{t}=\mathbf{6 0 s}$ & $\mathbf{4 6 5 . 7}$ & 476.1 & 509.3 & 917.2 \\
\hline $\mathbf{t}=\mathbf{6 0 0 s}$ & $\mathbf{4 6 4 3 . 2}$ & 4700.5 & 5077.6 & 9169.8 \\
\hline $\mathbf{t}=\mathbf{1 8 0 0 s}$ & $\mathbf{1 3 9 2 6 . 4}$ & 14088.2 & 15229.2 & 27508.7 \\
\hline $\mathbf{t}=\mathbf{3 6 0 0 s}$ & $\mathbf{2 7 8 5 1 . 2}$ & 28169.7 & 30456.6 & 55017.1 \\
\hline $\mathbf{t}=\mathbf{1 4 4 0 0}$ & $\mathbf{1 1 1 4 0 0 . 3}$ & 112658.5 & 121821.2 & 220067.6 \\
\hline $\mathbf{t}=\mathbf{2 8 8 0 0}$ & $\mathbf{2 2 2 7 9 8 . 9}$ & 225310.3 & 243640.6 & 440134.8 \\
\hline
\end{tabular}


Table 13: Time-cost effectiveness for applying PRIOSLICE variants, Weiser's slicing (WSlice) and thin slicing (Thin Slice) on the twelve faults that are applicable to thin slicing for each inspection time $t$ a programmer spends on each statement in the slice

\begin{tabular}{|l|r|r|r|r|r|}
\hline & PRIOSLICE $_{\text {lite }}$ & PRIOSLICE $_{\text {succ }}$ & PrIOSLICE $_{\text {update }}$ & WSlice & Thin Slice \\
\hline $\mathbf{t = 1 5 s}$ & $\mathbf{1 0 7 . 3}$ & 199.3 & 239.1 & 249.3 & 161.1 \\
\hline $\mathbf{t = 3 0 s}$ & $\mathbf{2 3 8 . 2}$ & 268.0 & 360.5 & 577.3 & 394.6 \\
\hline $\mathbf{t = 6 0 s}$ & 423.7 & 441.7 & 532.0 & 591.5 & $\mathbf{4 2 1 . 4}$ \\
\hline $\mathbf{t}=\mathbf{6 0 0 s}$ & 4220.9 & 4348.6 & 5301.4 & 5912.1 & $\mathbf{4 2 1 1 . 0}$ \\
\hline $\mathbf{t = 1 8 0 0 s}$ & 12659.2 & 13030.6 & 15900.2 & 17735.7 & $\mathbf{1 2 6 3 2 . 2}$ \\
\hline $\mathbf{t = 3 6 0 0 s}$ & 25316.6 & 26053.5 & 31798.3 & 35471.1 & $\mathbf{2 5 2 6 4 . 1}$ \\
\hline $\mathbf{t = 1 4 4 0 0 s}$ & 101261.0 & 104191.2 & 127187.0 & 141883.5 & $\mathbf{1 0 1 0 5 5 . 3}$ \\
\hline $\mathbf{t = 2 8 8 0 0 s}$ & 202520.1 & 208374.8 & 254372.0 & 283766.7 & $\mathbf{2 0 2 1 1 0 . 3}$ \\
\hline
\end{tabular}

SLICE $_{\text {succ }}$-but for the other four subjects the difference was larger. However, PRIOSLICE $_{\text {lite }}$ is, on average, 3.87 times faster than PRIOSLICE succ $_{\text {and }}$ an times

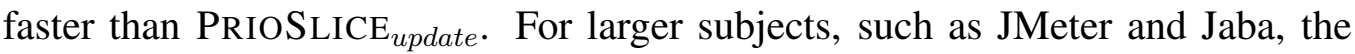

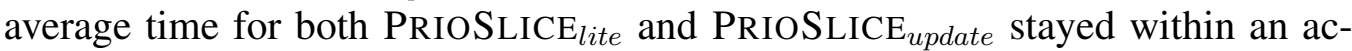
ceptable range (15 minutes). For the largest subject, PDFBox, the average time for PRIOSLICE increased considerably, but for two variants this time was still less than 40 minutes.

These numbers provide hints on how the two factors that define the variants of PRIOSLICE influenced its efficiency. The modest differences between PRIOSLICE $_{\text {lite }}$ and PRIOSLICE updating the probabilities in the priority queue during the traversal as new dependence paths are discovered. However, the much larger differences between

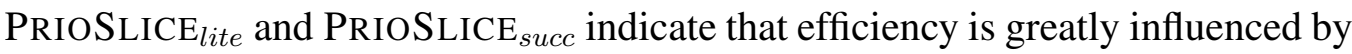
computing and propagating information from all dependence successors instead of only discovered successors during a traversal.

Table 10 and 11 present the time-cost effectiveness of applying each technique on all subjects when statement inspection time is set to 30 seconds. It is a ratio of time and effectiveness of the technique of which smaller value is better. "Not applicable" indicates that the techniques are not comparable for that subject because thin slicing failed to locate any of the faults of the subject. We use the term time-cost effectiveness to distinguish it from cost effectiveness in section 5.3.3 which measures the effectiveness per localization effort. To further study the effectiveness of each static slicing in fault localization tasks, we also measured the 
time-cost effectiveness assuming inspection time for each statement is, on average, 15 seconds, 1 minute (60 seconds), 10 minutes (600 seconds), 30 minutes (1800 seconds), 1 hours (3600 seconds), 4 hours (14400 seconds), and 8 hours (28800 seconds), shown in Table 12 and Table 13.

When inspection time for each statement is set to 30 seconds, for all faults, PRIOSLICE variants are more time-cost effective than Weiser's slicing, with the only exceptions for NanoXML, for which only PRIOSLICE update $_{\text {is }}$ better. Results shown in Table 10 are similar to those in Table 5, except that PRIOSLICE succ $_{\text {is }}$ the

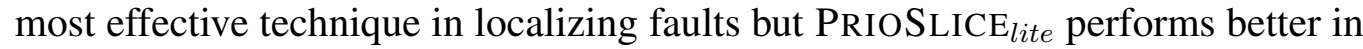
terms of time-cost effectiveness. For those faults that are applicable to thin slicing, we obtained the same observations for effectiveness and time-cost effectiveness that thin slicing only performs better in two cases and PRIOSLICE lite $_{\text {outperforms }}$ on average. These results indicate that even though it takes longer running times for PRIOSLICE variants but the time-cost effectiveness shows the strength of the techniques in localizing faults effectively and efficiently when statement inspection time is set to 30 seconds.

However, statement inspection time could vary widely due to different factors, for example, the complexity of the fault and the failure, programmer's expertise in the programming language, how familiar the programmer is to the application and so on. As far as we know, there is no comprehensive user study for average inspection time a programmer spends on deciding whether the statement is faulty or not. Therefore, we measured time-cost effectiveness for applying each static slicing technique for a range of statement inspection time, from 15 seconds to 8 hours. Table 12 shows that all PRIOSLICE variants outperform Weiser's static slicing for all statement inspection time by about $50 \%$, except when $t=30 \mathrm{~s}$ PRIOSLICE outperforms by about $60 \%$. Table 13 shows that for the twelve faults that thin slicing can apply, PRIOSLICE lite $_{\text {has }}$ the best time-cost effectiveness when $t=15 \mathrm{~s}$ and $t=30 \mathrm{~s}$. When the statement inspection time increases, thin slicing has a better time-cost effectiveness by $0.1 \%$ to $0.47 \%$ compared with the PRIOSLICE variant that has the best time-cost effectiveness. Results in Table 12 and Table 13 indicate that the time-cost effectiveness of applying each slicing technique to a fault localization task increases when the statement inspection time decreases. PRIOSLICE has a better time-cost effectiveness on average than Weiser's slicing, and performs slightly behind thin slicing when thin slicing can apply. To fundamentally further increase the time-cost effectiveness of PRIOSLICE, we would need to provide extra hints of a statement in the slice being faulty or not, which is not in the scope of this paper but in the plan of our future work.

These results, in conjunction with the effectiveness of the three variants of 
PRIOSLICE, suggest a trade off between their costs and benefits. A user who can afford to wait an hour or two should use PRIOSLICE succ to localize a fault slightly faster. If the user cannot wait that long, the two other variants of PRIOSLICE will provide almost the same benefits for only a fraction of that time. In all, we consider that these results validate the practicality of PRIOSLICE in terms of computational costs.

\subsection{Strength and Weakness}

The goal of PRIOSLICE is to improve the effectiveness of static slicing techniques in fault localization tasks by providing the developers more effective clues of where the faults may locate. In this section, we summarize the strength and weakness of PRIOSLICE in terms of when the effectiveness of PRIOSLICE benefits users more and when it benefits less in a fault localization task.

One strength of PRIOSLICE is that compared with other static slicing techniques, PRIOSLICE guarantees higher effectiveness on average. All PriOSLICE variants rank the faulty statement higher than Weiser's slicing on average, and perform similarly with thin slicing but have a larger applicability. Therefore, users could have a higher confidence in the suggested fault locations generated by PRIOSLICE. The second strength of PRIOSLICE is that it provides the users flexibility to choose between precision and cost. For the most precise result the user could

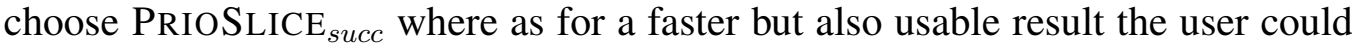
choose the other two variants of PRIOSLICE. The flexibility enables the users to run customized analysis on demand.

One weakness of PRIOSLICE is that when the shortest path between fault and failure is control-dependence dominated while there are many data-dependence dominated paths between non-faulty statements and the failing statement, Weiser's static slicing is a better choice than PRIOSLICE in terms of both effectiveness and efficiency. We suspect, though, that this particular case is rare considering all possible program structures, fault locations and failure locations, however we need to conduct further study to quantify the weakness.

Yet another weakness of PRIOSLICE is that when the faulty statement has a low propagation probability, the user would have to traverse a large portion of the slice until he reaches that statement. This happens usually when the distance between the faulty statement and failing statement is long. If the path is not control-dependence dominated, PRIOSLICE performs better than Weiser's static slicing and thin slicing and can provide a more effective suggestion of the fault location. If the path in control-dependence dominated, the problem goes back the first weakness discussed above. Due to the limitation of static slicing technique, 
the practical usability of the ranked list of fault locations needs to be improved when the fault has a low propagation probability.

Finally, one weakness of PRIOSLICE is that the effectiveness is based on the assumption of perfect bug understanding [26]. To minimize this weakness, we measured and compared time-cost effectiveness of PRIOSLICE with other static slicing techniques. The results show that the time-cost effectiveness increases when the statement inspection time decreases. In other words, to fundamentally increase the time-cost effectiveness of PRIOSLICE further more, we would need to decrease programmers' statement inspection time by providing more hints of whether the statement is faulty or not. Moreover, to fully access the usefulness of static slicing techniques, for future work, we plan to conduct user studies to learn how much users can benefit from static slicing techniques for fault localization tasks.

\subsection{Threats to Validity}

The main internal threat to the validity of the study is the possibility of implementation errors in PRIOSLICE or DUA-FORENSICS when slicing and computing the PRIOSLICE probabilities. However, this threat is mitigated by the maturity of DUA-FORENSICS, which has been developed for years and has been used in many other studies. We also tested PRIOSLICE carefully using examples and the studied subjects because this is the latest part of our code base. Another internal threat is the possibility of procedure errors in the use of PRIOSLICE or DUA-FORENSICS. To reduce this risk, we tested the results of each phase of the process.

The main external threat to this study is our sample of subjects and faults. The 35 faults we used in our study, however, span five subjects that are used in realworld scenarios. The faults are spread across these subjects and create different kinds of failures at various points in those subjects. These subjects exhibit various programming styles and have different purposes. These subjects have also been used in experiments conducted by other researchers in the past.

For construct validity, one threat is the way we measured and compared the effectiveness of PRIOSLICE and the other slicing techniques. Our study assumes that programmers benefit from static slicing and especially from ordered traversal of dependence graphs for static slicing. How much this metric reflects the real benefits can only be measured via user studies. However, the literature already provides hints that this is a more realistic representation of how developers debug than other approaches to fault localization [26].

Finally, a conclusion threat is the appropriateness of the statistical analysis and the data points we used. To control this threat, we used well-known non- 
parametric statistical tests which do not make assumptions about the distribution of the data [37]. Another aspect of this threat is the equal number of faults used for each subject, corresponding to the maximum number of faults available for most of these subjects. For consistency, we used the same number for all subjects. However, the complexity and uniqueness of each fault was approximately the same regardless of the size of the subject, which reduced this threat.

\section{Related Work}

Program slicing was introduced as an analysis for program comprehension and debugging [1,4]. Unfortunately, static slices are often too big to be useful. Our work alleviates this problem by recognizing that not all statements are equally relevant in a slice and that a static analysis can estimate their relative relevance to improve the effectiveness of the forward slice.

In preliminary work [29, 30], we described a forward version of probabilistic slicing which estimates the impacts of a change and creates a global ranking for inspecting impacted statements, regardless of their dependence distance from the slicing criterion. We have also investigated how to statically estimate the occurrence of data dependencies [31]. PRIOSLICE is our next iteration in this line of work and the first to operate backwards for tasks such as fault localization. Unlike previous work, PRIOSLICE uses probabilities to prioritize the traversal of dependence graphs, which mirrors what developers do [26, 16, 1], and we showed that this new approach can outperform other static-slicing approaches.

A few other techniques discriminate among statements within slices. Two of them work on dynamic slices to estimate the influence of statements on outputs $[38,17]$. These techniques, however, require runtime information which might not be readily available. Statically, thin slicing [16] distinguishes statements in slices by pruning control dependencies and pointer-based data dependencies, although these dependencies can be incorporated later. Our technique, in contrast, keeps all statements from the static slice from the very beginning, which is a safe approach, and instead estimates their influence to prioritize the traversal of backward slices.

In addition to the techniques mentioned above, dynamic slicing [5, 6] and other variants of program slicing have been proposed to reduce the size of slices. Conditioned slicing [15] lets users reduce slice sizes by restricting the input space, which is a feature PRIOSLICE currently does not provide. Researchers have also explored combining static slices with execution data, such as call stacks and dynamic aliasing $[10,11,12,13]$. Although PRIOSLICE is designed on purpose to 
avoid execution data, such data could be used to refine PRIOSLICE in future work.

Beyond slicing, there is a considerable amount of work on fault localization. One technique somewhat related to PRIOSLICE is the probabilistic program dependence graph [39], which uses a statistical model of the runtime behavior of individual dependencies. Statistical approaches, based on code coverage, have been proposed and widely studied (e.g., [18, 40, 41, 42]). Spectrum-based fault localization [40], for example, use execution profiles and similarity coefficients to rank potential fault locations. Improved statistical approaches mixed with slicing techniques (e.g., $[43,44]$ ) have been proposed to overcome the limitations of using only code coverage.

Execution differencing [3, 45] and delta debugging [46] are other well-known approaches. All these non-slicing approaches, however, require at least one test case to generate dynamic information, which makes these approaches essentially different from our technique. Our technique is purely static and requires only a report to identify a failing point in the program.

\section{Conclusion and Future Work}

In this paper, we presented PRIOSLICE, a new static-slicing technique that estimates the propagation probability of each statement in a static backward slice to indicate not only that the statement is in the slice, but also how much it is in that slice. These values are used by PRIOSLICE to traverse the dependence graph by decreasing order of the estimated influence of statements. We described our probabilistic model used to compute these values and justified our design decisions. We also created three variants of PRIOSLICE to study the cost-benefits of design choices for PRIOSLICE.

Our experimental results suggest that PRIOSLICE can, on average, spend less effort localizing faults than Weiser's original slicing approach and thin slicingeven in the cases that thin slicing was applicable. We also conducted statistics tests which show that in 4 out of 6 cases, we have confidence that our technique is more effective than the others.

This work provides a new way of looking at slices, in which statements are distinguished by relevance or strength. This work can be potentially used not only for fault localization, but also for other tasks such as program comprehension. Also, a forward version of this model could be used for change-impact analysis and test-case prioritization. Our probabilistic model can be further improved to potentially achieve an even greater level of effectiveness. 
For the near future, we plan to improve the precision of the model by using static branch-prediction heuristics (e.g., [47]) to more accurately model successorstatement probabilities in our model. We also intend to extend our studies to related applications such as program comprehension to assess the full extent of the benefits for developers of this novel approach to slicing. Moreover, we also plan to conduct user studies with real program developers to study 1) how much developers can benefit from using program slice for fault localization, and 2) how effective PRIOSLICE is compared with Weiser's slicing and thin slicing in helping developers localizing faults.

\section{Acknowledgments}

This work was supported in part by ONR award N00014-14-1-0037 to the University of Notre Dame. We also thank Haipeng Cai for his technical support and the late Mary Jean Harrold for her contributions to initiate this line of work.

[1] M. Weiser, Program slicing, IEEE Transactions on Software Engineering 10 (4) (1984) 352-357.

[2] D. Binkley, N. Gold, M. Harman, An Empirical Study of Static Program Slice Size, ACM Transactions on Software Engineering and Methodology $16(2)$.

[3] R. Santelices, M. J. Harrold, A. Orso, Precisely detecting runtime change interactions for evolving software, in: Proceedings of IEEE International Conference on Software Testing, Verification and Validation, 2010, pp. 429438.

[4] S. Horwitz, T. Reps, D. Binkley, Interprocedural Slicing Using Dependence Graphs, ACM Transactions on Program Languages and Systems, 12(1):2660.

[5] B. Korel, J. Laski, Dynamic Program Slicing, Information Processing Letters 29 (3) (1988) 155-163.

[6] H. Agrawal, J. R. Horgan, Dynamic Program Slicing, in: Proceedings of ACM Conference on Programming Language Design and Implementation, 1990, pp. 246-256. 
[7] A. Beszedes, C. Farago, Z. Mihaly Szabo, J. Csirik, T. Gyimothy, Union slices for program maintenance, in: Proceedings of IEEE International Conference on Software Maintenance, 2002, pp. 12-21.

[8] R. A. DeMillo, H. Pan, E. H. Spafford, Critical Slicing for Software Fault Localization, in: Proceedings of ACM International Symposium on Software Testing and Analysis, 1996, pp. 121-134.

[9] R. J. Hall, Automatic extraction of executable program subsets by simultaneous dynamic program slicing, Automated Software Engineering Journal 2 (1) (1995) 33-53.

[10] R. Gupta, M. L. Soffa, Hybrid Slicing: An Approach for Refining Static Slices Using Dynamic Information, in: Proceedings of ACM International Symposium on Foundations of Software Engineering, 1995, pp. 29-40.

[11] S. Horwitz, B. Liblit, M. Polishchuk, Better Debugging via Output Tracing and Callstack-sensitive Slicing, IEEE Transactions on Software Engineering 36 (1) (2010) 7-19.

[12] J. Krinke, Effects of Context on Program Slicing, Journal of Systems and Software 79 (9) (2006) 1249-1260.

[13] M. Mock, D. C. Atkinson, C. Chambers, S. J. Eggers, Program Slicing with Dynamic Points-to Sets, IEEE Transactions on Software Engineering 31 (8) (2005) 657-678.

[14] M. Acharya, B. Robinson, Practical Change Impact Analysis Based on Static Program Slicing for Industrial Software Systems, in: Proceedings of IEEE/ACM International Conference on Software Engineering, Software Engineering in Practice Track, 2011, pp. 746-765.

[15] G. Canfora, A. Cimitile, A. De Lucia, Conditioned Program Slicing, Information and Software Technology 40 (11-12) (1998) 595-608.

[16] M. Sridharan, S. J. Fink, R. Bodik, Thin slicing, in: Proceedings of ACM Conference on Programming Language Design and Implementation, 2007, pp. 112-122.

[17] X. Zhang, N. Gupta, R. Gupta, Pruning Dynamic Slices with Confidence, in: Proceedings of ACM Conference on Programming Language Design and Implementation, 2006, pp. 169-180. 
[18] J. A. Jones, M. J. Harrold, J. Stasko, Visualization of Test Information to Assist Fault Localization, in: Proceedings of IEEE/ACM International Conference on Software Engineering, 2002, pp. 467-477.

[19] J. A. Jones, M. J. Harrold, Empirical Evaluation of the Tarantula Automatic Fault-Localization Technique, in: Proceedings of IEEE/ACM International Conference on Automated Software Engineering, 2005, pp. 273-282.

[20] A. V. Aho, M. Lam, R. Sethi, J. D. Ullman, Compilers: Principles, Techniques and Tools, Prentice Hall, 2006.

[21] J. Ferrante, K. J. Ottenstein, J. D. Warren, The Program Dependence Graph and Its Use in Optimization, ACM Transactions on Program Languages and Systems, 9(3):319-349.

[22] L. O. Andersen, Program analysis and specialization for the $\mathrm{C}$ programming language, Ph.D. Thesis, DIKU, U. of Copenhagen (May 1994).

[23] W. Masri, A. Podgurski, Measuring the Strength of Information Flows in Programs, ACM Transactions on Software Engineering and Methodology 19 (2) (2009) 1-33.

[24] G. Ramalingam, Data Flow Frequency Analysis, in: Proceedings of ACM Conference on Programming Language Design and Implementation, 1996, pp. 267-277.

[25] J. Pearl, Heuristics: Intelligent Search Strategies for Computer Problem Solving, Addison-Wesley, 1984.

[26] C. Parnin, A. Orso, Are Automated Debugging Techniques Actually Helping Programmers?, in: Proceedings of ACM International Symposium on Software Testing and Analysis, 2011, pp. 199-209.

[27] R. Santelices, M. J. Harrold, Efficiently Monitoring Data-flow Test Coverage, in: Proceedings of IEEE/ACM International Conference on Automated Software Engineering, 2007, pp. 343-352.

[28] R. Santelices, Y. Zhang, H. Cai, S. Jiang, DUA-Forensics: A Fine-Grained Dependence Analysis and Instrumentation Framework Based on Soot, in: Proceedings of ACM SIGPLAN Int'1 Workshop on the State Of the Art in Java Program Analysis, 2013, pp. 13-18. 
[29] R. Santelices, M. J. Harrold, Probabilistic slicing for predictive impact analysis, Techical Report CERCS-10-10, Georgia Tech (Nov. 2010).

[30] R. Santelices, Y. Zhang, S. Jiang, H. Cai, Y. jie Zhang, Quantitative Program Slicing: Separating Statements by Relevance, in: Proc. of Int'l Conf. on Softw. Eng., New Ideas and Emerging Results, 2013, pp. 1269-1272.

[31] Y. Zhang, R. Santelices, Predicting Data Dependences for Slice Inspection Prioritization, in: Proceedings of IEEE International Workshop on Program Debugging, 2012, pp. 177-182.

[32] P. Jaccard, Étude Comparative de la Distribution Florale dans une Portion des Alpes et des Jura, Bulletin de la Société Vaudoise des Sciences Naturelles 37 (1901) 547-579.

[33] G. Boole, An Investigation of the Laws of Thought: On which are Founded the Mathematical Theories of Logic and Probabilities, Walton and Maberly, 1854.

[34] H. Do, S. Elbaum, G. Rothermel, Supporting Controlled Experimentation with Testing Techniques: An Infrastructure and its Potential Impact, Empirical Software Engineering 10 (4) (2005) 405-435.

[35] JABA: Java Architecture for Bytecode Analysis, http://pleuma.cc . gatech.edu/aristotle/Tools/jaba.html, georgia Institute of Technology, 2005.

[36] A. J. Offutt, A. Lee, G. Rothermel, R. H. Untch, C. Zapt, An experimental determination of sufficient mutant operators, ACM Transcations on Software Engineering and Methodology 5 (1996) 99-118.

[37] R. E. Walpole, R. H. Myers, S. L. Myers, K. E. Ye, Probability and Statistics for Engineers and Scientists, Prentice Hall, 2011.

[38] T. Goradia, Dynamic Impact Analysis: A Cost-effective Technique to Enforce Error-propagation, in: Proceedings of ACM International Symposium on Software Testing and Analysis, 1993, pp. 171-181.

[39] G. K. Baah, A. Podgurski, M. J. Harrold, The Probabilistic Program Dependence Graph and Its Application to Fault Diagnosis, IEEE Transactions on Software Engineering 36 (4) (2010) 528-545. 
[40] R. Abreu, P. Zoeteweij, A. J. C. V. Gemund, On the Accuracy of Spectrumbased Fault Localization, in: Proceedings of Testing: Academic and Industrial Conference - Practice and Research Techniques, 2007, pp. 89-98.

[41] R. Santelices, J. A. Jones, Y. Yu, M. J. Harrold, Lightweight Fault Localization Using Multiple Coverage Types, in: Proceedings of IEEE/ACM International Conference on Software Engineering, 2009, pp. 56-66.

[42] V. Debroy, W. E. Wong, A Consensus-based Strategy to Improve the Quality of Fault Localization, Software: Practice and Experience, published online November 43 (8) (2011) 989-1011. doi : 10 . 1002 / spe. 1146.

[43] Y. Lei, X. Mao, T. Y. Chen, Backward-slice-based statistical fault localization without test oracles, in: Proceedings of International Conference on Quality Software, 2013, pp. 212-221.

[44] X. Mao, Y. Lei, Z. Dai, Y. Qi, C. Wang, Slice-based statistical fault localization, The journal of Systems and Software 89 (2014) 51-62.

[45] W. N. Sumner, X. Zhang, Comparative Causality: Explaining the Differences Between Executions, in: Proceedings of IEEE/ACM International Conference on Software Engineering, 2013, to appear.

[46] A. Zeller, Why Programs Fail: A Guide to Systematic Debugging, 2nd Edition, Morgan Kaufmann, 2009.

[47] T. Ball, J. R. Larus, Branch Prediction for Free, in: Proceedings of ACM Conference on Programming Language Design and Implementation, 1993, pp. 300-313. 